\title{
A coagulation-fragmentation model for the turbulent growth and destruction of preplanetesimals
}

\author{
A. Johansen, F. Brauer, C. Dullemond, H. Klahr, and T. Henning
}

\author{
Max-Planck-Institut für Astronomie, Königstuhl 17, 69117 Heidelberg, Germany \\ e-mail: johansen@mpia.de
}

Received 11 December 2007 / Accepted 3 April 2008

\begin{abstract}
To treat the problem of growing protoplanetary disc solids across the meter barrier, we consider a very simplified two-component coagulation-fragmentation model that consists of macroscopic boulders and smaller dust grains, the latter being the result of catastrophic collisions between the boulders. Boulders in turn increase their radii by sweeping up the dust fragments. An analytical solution of the dynamical equations predicts that growth by coagulation-fragmentation can be efficient and allow agglomeration of 10-m-sized objects within the time-scale of the radial drift. These results are supported by computer simulations of the motion of boulders and fragments in 3-D time-dependent magnetorotational turbulence. However allowing the fragments to diffuse freely out of the sedimentary layer of boulders drastically reduces the density of both boulders and fragments in the mid-plane, and thus also the growth of the boulder radius. The reason is that the turbulent diffusion time-scale is so much shorter than the collisional time-scale that dust fragments leak out of the mid-plane layer before they can be swept up by the boulders there. Our conclusion that coagulationfragmentation is not an efficient way to grow across the meter barrier in fully turbulent protoplanetary discs confirms recent results by Brauer, Dullemond, \& Henning who solved the coagulation equation in a parameterised turbulence model with collisional fragmentation, cratering, radial drift, and a range of particle sizes. We find that a relatively small population of boulders in a sedimentary mid-plane layer can populate the entire vertical extent of the disc with small grains and that these grains are not first generation dust, but have been through several agglomeration-destruction cycles during the simulations.
\end{abstract}

Key words. accretion, accretion disks - stars: planetary systems: formation - stars: planetary systems: protoplanetary disks solar system: formation - turbulence

\section{Introduction}

Explaining the formation of km-sized planetesimals from $\mu \mathrm{m}$-sized dust grains is a long-standing challenge for planet formation models. The problem is complicated by the interplay of an array of different physics - most notably the turbulence of protoplanetary discs and the sticking and collisional destruction of solids of different sizes (Chokshi et al. 1993; Weidenschilling \& Cuzzi 1993; Dominik \& Tielens 1997; Blum \& Wurm 2000; Henning et al. 2006).

Young stars are known to receive mass from the inner part of their circumstellar disc (Bertout et al. 1988). The cause of such accretion is most likely that protoplanetary discs are turbulent. The source of turbulence must in this connection be a Keplerian shear instability (such as magnetorotational instability, see Balbus \& Hawley 1998) or self-gravity if the disc is massive enough (Balbus \& Papaloizou 1999; Gammie 2001; Lodato \& Rice 2004). There are nevertheless significant problems with both these sources of accretion: the ionisation fraction of protoplanetary discs at 1-20 AU from the star may be too low for the magnetorotational instability to operate (Gammie 1996; Semenov et al. 2004), while only a minor fraction of discs is expected to be massive enough to be gravitationally unstable (Beckwith et al. 1990). Thus turbulence is often treated as a free parameter in protoplanetary disc models, parameterised through an $\alpha$-value (i.e. turbulent viscosity) anywhere from $\alpha=10^{-6}$ up to as high as $\alpha=0.1$.

Collisions between $\mu \mathrm{m}$-sized dust monomers leads to the formation of dust aggregates under a range of conditions
(Blum \& Wurm 2000). But larger bodies have poor sticking properties and a lower threshold for collisional destruction (Chokshi et al. 1993; Benz 2000). The sticking problem is especially acute for m-sized boulders. The strength of these bodies is very low, while collision speeds, induced by the turbulent gas and by the differential settling and radial drift, are high (Völk et al. 1980; Mizuno et al. 1988; Weidenschilling 1997). The slightly sub-Keplerian gas acts as a constant head wind on the boulders, draining them of angular momentum and causing them to drift radially through the disc (Weidenschilling 1977). The drift rate is approximately proportional to the radius of the boulders, introducing a differential radial drift that can be as strong as $50 \mathrm{~m} / \mathrm{s}$ between bodies of $1 \mathrm{~m}$ and $10 \mathrm{~cm}$ in size.

Modelling the growth of solids in protoplanetary discs requires solution of the coagulation equation (or Smoluchowski equation) that governs the time evolution of a size distribution of solids. Pioneering work on the numerical solution of the coagulation equation in a planetesimal formation context was done by Weidenschilling (1984) who found that particle growth in turbulent discs is efficient because the relative speeds induced by the turbulence give high collision rates. Collisional fragmentation nevertheless halts growth when the solids reach sizes of a few cm (pebbles). These early models were improved in Weidenschilling (1997) to include many more bins in the vertical direction and in the particle radius. Considering the formation of comets at $30 \mathrm{AU}$ from the proto-Sun, Weidenschilling concluded that coagulation could in principle explain the growth all the way to $\mathrm{km}$-sized planetesimals, although for disc 
models where the turbulence is purely induced by the sedimentation, so that very high particle densities occur in the mid-plane. The sticking efficiency was also assumed to be high, in some contrast with later models of boulder collisions (Benz 2000; Schäfer et al. 2007), and collisional fragmentation was kept at a minimum by assuming a constant specific kinetic energy threshold for fragmentation. In this approach bodies of different sizes may collide at very high speeds without destroying each other (see Appendix F of Weidenschilling 1997).

Dullemond \& Dominik (2005) presented simulations similar to Weidenschilling (1997) and found numerically that the size distribution of solids can arrive at a balance between collisional fragmentation and coagulation. The first simulations to include the full radial extent of a protoplanetary disc were presented recently by Brauer et al. (2008, hereafter BDH). BDH found that the meter barrier is a genuine problem for planetesimal formation, both because macroscopic bodies destroy each other in catastrophic collisions and because radial drift sends macroscopic bodies into the inner nebula where they are lost from the planet formation process. Radial drift is not only a problem for m-sized boulders: over the life-times of protoplanetary discs (millions of years, see e.g. Bouwman et al. 2006) even mmand $\mathrm{cm}$-sized pebbles have significant drift and are emptied from the outer parts of the nebula (Takeuchi \& Lin 2002; Brauer et al. 2007).

In this paper we isolate the effect of collisional fragmentation and subsequent sweep-up of fragments on the growth of boulders. We do not solve the full coagulation equation as in $\mathrm{BDH}$, but simplify the size distribution to effectively two bins small dust fragments and large boulders - in order to make the particle growth tractable in a 3-D simulation with magnetised time-dependent turbulence.

The paper is structured as follows. In Sect. 2 we describe our simplified two-component model of boulders and fragments in detail and find an equilibrium solution of the dynamical equations. In Sect. 3 we describe the numerical simulations that will be used to evolve the dynamical equations. A local corotating box is considered, and turbulence is produced by the magnetorotational instability. Dust fragments are treated as a passive scalar, while the boulders are treated as individual superparticles with two internal degrees of freedom: the number density of actual particles inside each superparticle and the average radius of the constituent boulders. In the next section, Sect. 4, we present simulations where the dust fragments are not allowed to leave the boulder layer. The system quickly evolves towards the equilibrium state found in Sect. 2 with rapid growth of the boulders of a few mm per orbit. In Sect. 5 we briefly turn to the analytical model again and see how the diffusion of dust fragments from the boulder layer affects the equilibrium solution. The turbulent gas transports fragments out of the mid-plane, and thus the growth rate of the boulders is reduced. This is confirmed in computer simulations where boulders lie in a thin layer around the mid-plane, presented in Sect. 6. The dust fragments spread quickly out of the mid-plane and the growth rate of the boulders is reduced by a factor of 10 , so that the growth can no longer compete with the radial drift. The whole disc is filled with dust fragments that are formed in the thin mid-plane layer. We conclude on our results in Sect. 7 and speculate about ways to overcome coagulation-fragmentation growth barriers. We find that radial drift, not collisional fragmentation, is the more serious problem for coagulation-fragmentation growth and discuss processes to reduce or stop radial drift in actual protoplanetary discs.

\section{Coagulation-fragmentation model}

We consider a simple two-species model of solids in a protoplanetary disc. Species 1 consists of tiny dust grains with mass $m_{1}$ and number density $n_{1}$, while species 2 consists of macroscopic boulders with mass $m_{2}$ and number density $n_{2}$. Here "dust grains" are defined as being so small that they couple to the gas on a time-scale that is much shorter than an orbital time, while "boulders" are solid bodies with sizes between $10 \mathrm{~cm}$ and $10 \mathrm{~m}$.

We assume for simplicity that

1. collisions between the tiny grains are insignificant compared to the sweep-up of the grains by the boulders;

2. collisions between a boulder and a dust grain always lead to the incorporation of the grain into the boulder;

3. collisions between the boulders lead to a complete destruction of the colliding bodies. The entire mass then ends up in tiny grains.

Thus we do not treat the problem of how to form boulders in the first place, but focus on how they grow by sweeping up dust. We also ignore effects like cratering in our treatment of boulder-dust collisions. Although the actual growth of boulders across the metre-barrier will likely involve a combination of many different aspects of collision physics (and also self-gravity), in this paper we will instead investigate the pure problem of collisional fragmentation of equal-sized boulders and sweep-up of small dust grains in a turbulent environment. Assuming an impact strength of zero for the boulders and perfect sticking between boulders and dust grains may not be entirely realistic, but this allows us to simplify our model greatly.

We can write dynamical equations for the number densities of dust and boulders and for the mass of the individual boulders,

$\frac{\partial n_{1}}{\partial t}=-n_{1} n_{2} \sigma_{12} v_{12}+n_{2}^{2} \sigma_{22} v_{22} \frac{m_{2}}{m_{1}}$

$\frac{\partial n_{2}}{\partial t}=-n_{2}^{2} \sigma_{22} v_{22}$

$\frac{\partial m_{2}}{\partial t}=\sigma_{12} v_{12} \rho_{1}$

Here $\sigma_{12}$ and $\sigma_{22}$ are the collisional cross sections for bouldergrain and boulder-boulder collisions, respectively, while $v_{12}$ and $v_{22}$ are the corresponding collision speeds. We furthermore introduced the bulk density of dust fragments $\rho_{1}=n_{1} m_{1}$. We assume next that the grains and the boulders are spheres with radius $a_{1}$ and $a_{2}$, respectively, and that $a_{1} \ll a_{2}$. The dynamical equation for $m_{2}$ can then be turned into a dynamical equation for the radius $a_{2}$,

$\frac{\partial a_{2}}{\partial t}=\frac{\rho_{1}}{4 \rho_{\bullet}} v_{12}$

with $\rho_{\bullet}$ referring to the material density of the solids.

\subsection{Equilibrium limit}

Equation (1) has the equilibrium solution

$\frac{\rho_{1}}{\rho_{2}}=4 \frac{v_{22}}{v_{12}}$

where we define the bulk densities ${ }^{1} \rho_{1}=n_{1} m_{1}, \rho_{2}=n_{2} m_{2}$ and set $\sigma_{22} \approx 4 \sigma_{12}$ under the assumption that the contribution of the

\footnotetext{
1 We use the term "bulk density" throughout this paper to refer to the total mass of solid material in a given volume divided by the volume, i.e. including the void between the solids.
} 


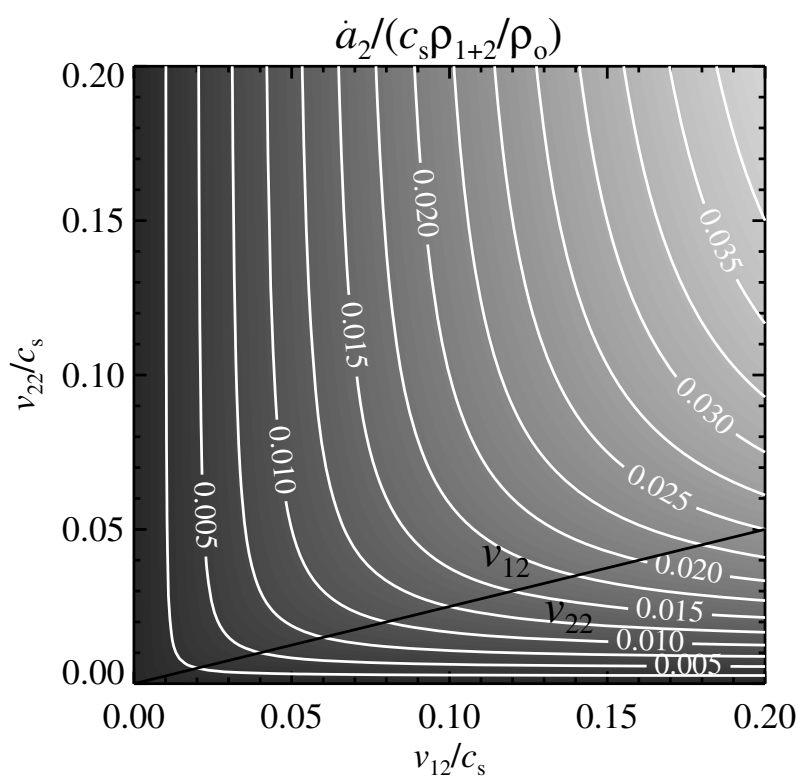

Fig. 1. Contour lines of the radius growth of boulders, $\dot{a}_{2}$, as a function of the collision speed between boulders and fragments, $v_{12}$, and between boulders and boulders, $v_{22}$. Two regimes are divided by the black line: for $v_{22} / v_{12} \ll 1 / 4$ the radius growth depends only on $v_{22}$, whereas the radius growth depends only on $v_{12}$ in the limit $v_{22} / v_{12} \gg 1 / 4$. Note that the normalisation of collision speeds to the sound speed is an arbitrary choice.

small grains to the cross section $\sigma_{12}$ is vanishingly small. We have also assumed, by setting the collisional radius of a boulder to twice its physical radius, that all impact parameters lead to destruction, even if the boulders collide at a small grazing angle. Thus if $v_{12} \approx v_{22}$ the system tends towards an equilibrium where the small grains in total contain four times more mass than the boulders. We show in Appendix A that any perturbation to the equilibrium solution will decay on a collisional time-scale, so that Eq. (5) constitutes a (both linearly and non-linearly) stable solution to the coagulation-fragmentation problem (Eq. (1)).

Inserting Eq. (5) into (4) yields the evolution of the boulder radius in the equilibrium state as

$\frac{\partial a_{2}}{\partial t}=\frac{\rho_{1+2}}{\rho_{\bullet}} \frac{v_{22}}{1+4 v_{22} / v_{12}}$.

Here we have introduced the bulk density of solids $\rho_{1+2}=\rho_{1}+$ $\rho_{2}$ which is constant in time in the absence of evaporation and condensation processes. We show in Fig. 1 the dependence of the radius growth on the collision speeds $v_{12}$ and $v_{22}$. It is clear from Eq. (6) that the radius growth depends only on either $v_{12}$ or $v_{22}$ at the two limits of $v_{22} / v_{12}$ (i.e. zero and infinity). The dividing line at $v_{22} / v_{12}=1 / 4$ is indicated with a black line in Fig. 1 .

Equation (6) implies that there is a linear growth of the boulder radius with time, although only under the assumption that $\rho_{1+2}, v_{12}$ and $v_{22}$ are independent of particle size (we shall include the full dependence of these parameters on the particle size in the numerical simulations presented in Sects. 3 and 6). For typical values of $\rho_{1+2} / \rho_{\bullet}=10^{-11}, v_{12}=25 \mathrm{~m} \mathrm{~s}^{-1}$ and $v_{22}=$ $10 \mathrm{~m} \mathrm{~s}^{-1}$, relevant in a sedimentary mid-plane layer of solids at $r=5$ AU of a moderately turbulent minimum mass solar nebula model with a turbulent viscosity of $\alpha=10^{-3}$ (see Sect. 3, for a definition of $\alpha$ ), the growth rate is $\dot{a}_{2}=4 \times 10^{-11} \mathrm{~m} \mathrm{~s}^{-1}$, or $1.2 \mathrm{~mm}$ per year. Around 2000 years are then needed to grow from $30 \mathrm{~cm}$, the size for which radial drift is the fastest, to $3 \mathrm{~m}$ in radius, which is so loosely coupled to the gas that radial drift is no longer a problem. In the absence of collisional fragmentation, on the other hand, the sweep-up will end after the boulders have incorporated all the small grains. Considering a fixed number density of boulders $n_{2}$, we can write the ratio of the particle radii $a_{2}$ and $a_{2}^{\prime}$ for two different mass densities $\rho_{2}$ and $\rho_{2}^{\prime}$ as

$$
\left(\frac{a_{2}^{\prime}}{a_{2}}\right)^{3}=\frac{\rho_{2}^{\prime}}{\rho_{2}} \text {. }
$$

Setting $\rho_{2}^{\prime}=\rho_{1}+\rho_{2}$, it is seen from Eq. (7) that if $4 / 5$ of the dust mass is originally in small grains, then the sweep-up of those grains by the boulders can only lead to a moderate increase of approximately $70 \%$ in the average boulder radius. Only when collisional fragmentation is included can the boulders grow larger than that, because the reservoir of grains to sweep up will be continuously replenished.

For the case $v_{12} \approx v_{22}$, Eq. (6) simplifies to

$\frac{\partial a_{2}}{\partial t}=\frac{1}{5} \frac{\rho_{1+2}}{\rho_{\bullet}} v_{22}$.

One can consider yet another special case of Eq. (6) where the collisions between boulders and grains happen at a much higher speed than the collisions between boulders and boulders, $v_{12} \gg$ $v_{22}$. This is relevant if the boulders migrate radially inwards due to a radial pressure gradient in the gas (Weidenschilling 1977). The drift speed can approach $10 \%$ of the sound speed, easily an order of magnitude higher than the turbulent gas motion that causes the collisions between boulders. Thus sweep-up works much more efficiently than fragmentation, and Eq. (6) changes to

$\frac{\partial a_{2}}{\partial t}=\frac{\rho_{1+2}}{\rho_{\bullet}} v_{22}$

which is five times faster than Eq. (8). The approximation that $v_{22} \ll v_{12}$ may nevertheless be unachievable, even if turbulent motion is weak, since shape effects will induce differential radial drift even between equal-mass bodies (Benz 2000).

\subsection{Timescale to reach equilibrium}

Starting from a state where an equal amount of mass is present in dust and in boulders, the coagulation-fragmentation equilibrium is reached when a significant fraction of the boulders have undergone collisions (and subsequent fragmentation). From Eq. (2) the time it takes to reach equilibrium $t_{\mathrm{eq}}$ is given by

$\frac{1}{t_{\mathrm{eq}}}=n_{2} \sigma_{22} v_{22}$.

Assuming that the boulders are spherical, the number density $n_{2}$ and the collisional cross section $\sigma_{22}$ can be written in terms of the solid radius $a_{2}$, yielding

$t_{\mathrm{eq}}=\frac{\rho_{\bullet} a_{2}}{3 \rho_{2} v_{22}}$.

When $a_{2}<(9 / 4) \lambda$, where $\lambda$ is the mean free path of the gas, the friction force is in the Epstein regime (see Appendix B for a discussion of the validity of the Epstein regime for the boulders). Here the friction time can be written as

$\tau_{\mathrm{f}}=\frac{a_{2} \rho_{\bullet}}{c_{\mathrm{s}} \rho_{\mathrm{g}}}$,

with $\rho_{\mathrm{g}}$ denoting the gas density and $c_{\mathrm{s}}$ the sound speed. Inserting this expression for the friction time in Eq. (11) yields

$\Omega_{\mathrm{K}} t_{\mathrm{eq}}=\frac{\Omega_{\mathrm{K}} \tau_{\mathrm{f}}}{3 \epsilon_{2} v_{22} / c_{\mathrm{s}}}$ 


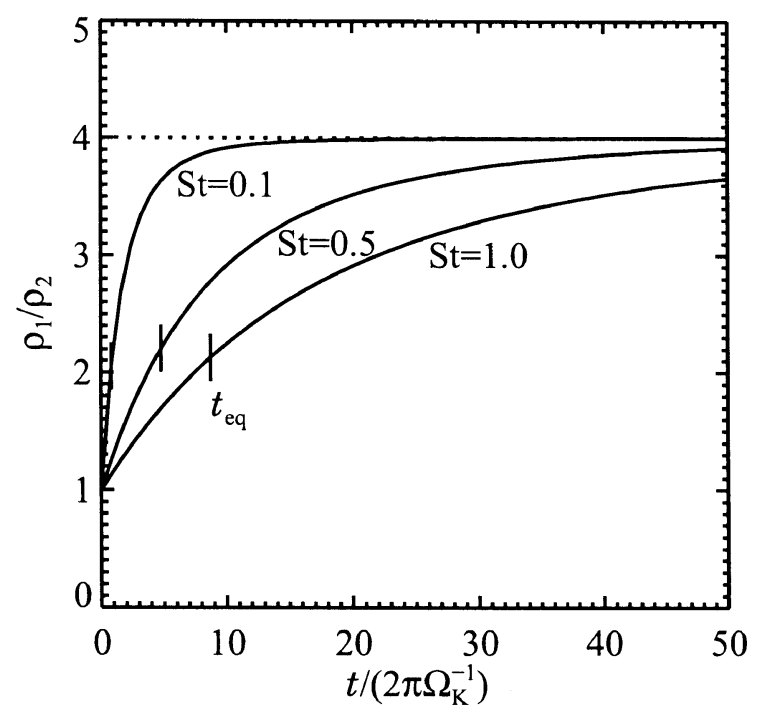

Fig. 2. The time evolution of the mass density in dust grains, $\rho_{1}$, relative to that of boulders, $\rho_{2}$. We have assumed an initial dust-to-gas ratio of 0.3 for both dust and boulders, collision speeds of $v_{12}=v_{22}=0.02 c_{\mathrm{s}}$ and a solid density of $\rho_{\bullet} / \rho_{\mathrm{g}}=10^{11}$. The timescale $t_{\mathrm{eq}}$ for approaching the equilibrium value $\rho_{1} / \rho_{2}=4$ (dashed line) is indicated with vertical lines.

Here $\Omega_{\mathrm{K}}=\Omega_{\mathrm{K}}(r)$ is the Keplerian angular frequency of the disc, a measure of the local dynamical time-scale at a given radial location in the disc, and $\epsilon_{2}$ is the ratio of the bulk densities of boulders and gas. We define a dimensionless friction time through the Stokes number St as

$\mathrm{St}=\Omega_{\mathrm{K}} \tau_{\mathrm{f}}$.

Thus the time it takes to reach a coagulation-fragmentation equilibrium is independent of the actual density of the surrounding gas. For a given Stokes number, any decrease in the gas density must be balanced by a similar decrease in the radius of the boulders, leading to an increase in the number density that balances the decrease in collisional cross section in Eq. (10). The presence of the sound speed in Eq. (13) also does not affect the timescale, since the turbulent collision speed $v_{22}$ must scale with the sound speed as well ${ }^{2}$.

The time evolution of the mass ratio $\rho_{1} / \rho_{2}$ between dust grains and boulders is shown in Fig. 2. We have integrated the 0-D coagulation-fragmentation equations (Eqs. (1), (2) and (4)) with an initial dust-to-gas ratio of 0.3 for both species (relevant in a mid-plane layer of solids in equilibrium between sedimentation and turbulent diffusion), collision speeds of $v_{12}=$ $v_{22}=0.02 c_{\mathrm{s}}$ and a solid density of $\rho_{\bullet} / \rho_{\mathrm{g}}=10^{11}$. The approach to the equilibrium state $\rho_{1} / \rho_{2}=4$ happens on the equilibrium timescale $t_{\text {eq }}$, given by Eq. (11). For $\mathrm{St}=0.5$ the equilibrium timescale is around five orbits, increasing proportionally to the Stokes number. The onset of fragmentation should depend on the radius of the boulders rather than on the Stokes number, so the critical Stokes number for which collisional fragmentation becomes important depends on the radial location in the disc. Around the location of Jupiter in a minimum mass nebula a particle with Stokes number unity has a radius of approximately $30 \mathrm{~cm}$ (see Sect. 3.4), for which collisional fragmentation should already be substantial. Since

\footnotetext{
2 This is strictly not the case in the presence of magnetic fields, where the local Alfvén speed gives a second velocity scale, but we shall ignore that complication here.
}

the coagulation-fragmentation equilibrium is stable (see Appendix A), the state will stay in equilibrium once it is reached.

It is not strictly necessary to be near the equilibrium value of $\rho_{1} / \rho_{2}$ to have efficient growth of the boulders by sweep-up. The radius of the boulders grows proportionally to $\rho_{1}$, according to Eq. (4), so just maintaining the reservoir of grains is an important achievement of the collisional fragmentation. As $\rho_{1}$ increases with time, the boulders will grow faster and faster until they reach the growth speed given by Eq. (6).

\section{Simulation set up}

Next we will solve the coagulation-fragmentation equations numerically in a three-dimensional time-dependent turbulent flow. In this section we describe the dynamical equations and the adopted protoplanetary disc model. We let turbulence arise through the magnetorotational instability (Balbus \& Hawley 1991) which operates when the gas is sufficiently ionised (Gammie 1996; Semenov et al. 2004). Typically the most unstable wavelength of the magnetorotational instability is around one gas scale height, with a subsequent energy cascade to smaller scales that approximately obeys a Kolmogorov-law (Hawley et al. 1995). The saturated state of the magnetorotational instability (which we will refer to as magnetorotational turbulence) is characterised by an outwards transport of angular momentum through positive Reynolds and Maxwell stresses. In shearing box simulations the measured $\alpha$-value ranges from $10^{-3}$ (with zero net flux field) to above 0.1 (for $\beta=P_{\text {gas }} / P_{\text {mag }}=400$ net vertical field, see Hawley et al. 1995). Numerically, magnetorotational turbulence has the great advantage that it is relatively easy to produce and sustain in local box simulations for hundreds of disc rotation periods (Brandenburg et al. 1995; Hawley et al. 1995).

Gullbring et al. (1998) and more recently Sicilia-Aguilar et al. (2004) measured the accretion luminosities of T Tauri stars and translated the measurements into a mass accretion rate $\dot{M}$. Typical estimated values of the mass accretion rate lie in the interval $\dot{M}=10^{-9 \ldots-7} M_{\odot} \mathrm{yr}^{-1}$. Coupling the mass accretion rate with a disc model yields the turbulent viscosity of the disc, $v_{\mathrm{t}}$, through the relation (Pringle 1981)

$v_{\mathrm{t}}=(3 \pi)^{-1} \frac{\dot{M}}{\Sigma}$

where $\Sigma$ is the column density of gas and solids. Making use of the non-dimensionalisation with sound speed $c_{\mathrm{s}}$ and angular frequency $\Omega_{\mathrm{K}}$ of Shakura \& Sunyaev (1973), $v_{\mathrm{t}}=\alpha c_{\mathrm{S}}^{2} \Omega_{\mathrm{K}}^{-1}$, we obtain the $\alpha$-value of the disc through

$\alpha=(3 \pi)^{-1} \frac{\dot{M}}{\Sigma} \frac{\Omega_{\mathrm{K}}}{c_{\mathrm{S}}^{2}}$.

For the minimum mass solar nebula $\alpha=10^{-4 \ldots-2}$ from typical mass accretion rates. The turbulent viscosity can be approximated as

$v_{\mathrm{t}}=\tau_{\text {eddy }} u_{\mathrm{rms}}^{2}$,

where $\tau_{\text {eddy }}$ is the eddy turn over time and $u_{\mathrm{rms}}$ is the turbulent rms speed. Assuming $\tau_{\text {eddy }} \approx \Omega_{\mathrm{K}}^{-1}$, due to the dominating effect of the Coriolis force at large scales (Weidenschilling 1984), one obtains $\alpha=\left(u_{\mathrm{rms}} / c_{\mathrm{s}}\right)^{2}$. However one must be careful when translating $\alpha$ into $u_{\mathrm{rms}}$ this way, since $\alpha$ normally refers to the turbulence's ability to diffuse the main Keplerian differential rotation, and instabilities that are not Keplerian shear instabilities are often associated with a negative $\alpha$-value (such as 
Table 1. Simulation parameters. The box size is fixed at $(1.32 H)^{3}$ in all runs. The initial column densities $\Sigma_{1}$ and $\Sigma_{2}$ are set in runs A and B to mimic the density in a sedimentary mid-plane layer; in the simulations with vertical gravity on the boulders (runs C-F), where dust fragments can leak freely out of the boulder layer, we set the column densities to a more canonical value of 0.01 for each component.

\begin{tabular}{cccccccccc}
\hline \hline Run & Resolution & Particles & Leaking & $\rho_{\bullet} / \rho_{\mathrm{g}}$ & $\Sigma_{1} / \Sigma_{\mathrm{g}}$ & $\Sigma_{2} / \Sigma_{\mathrm{g}}$ & $a_{2} / H$ & $\alpha$ & Simulation time \\
\hline $\mathrm{A}$ & $64^{3}$ & $2.0 \times 10^{6}$ & No & $10^{11}$ & 0.3 & 0.3 & $10^{-11}$ & $10^{-3}$ & $200 T_{\text {orb }}$ \\
$\mathrm{B}$ & $64^{3}$ & $2.0 \times 10^{6}$ & No & $10^{11}$ & 0.3 & 0.3 & $10^{-11}$ & $10^{-2}$ & $200 T_{\text {orb }}$ \\
$\mathrm{C}$ & $64^{3}$ & $2.5 \times 10^{5}$ & Yes & $10^{11}$ & 0.01 & 0.01 & $10^{-11}$ & $10^{-3}$ & $400 T_{\text {orb }}$ \\
$\mathrm{D}$ & $64^{3}$ & $2.5 \times 10^{5}$ & Yes & $10^{11}$ & 0.01 & 0.01 & $10^{-11}$ & $10^{-2}$ & $400 T_{\text {orb }}$ \\
$\mathrm{E}$ & $128^{3}$ & $2.0 \times 10^{6}$ & Yes & $10^{11}$ & 0.01 & 0.01 & $10^{-11}$ & $10^{-3}$ & $200 T_{\text {orb }}$ \\
$\mathrm{F}$ & $128^{3}$ & $2.0 \times 10^{6}$ & Yes & $10^{11}$ & 0.01 & 0.01 & $10^{-11}$ & $10^{-2}$ & $200 T_{\text {orb }}$ \\
\hline
\end{tabular}

convection or streaming instability, see Ryu \& Goodman 1992; Youdin \& Goodman 2005). For magnetorotational turbulence, Eq. (17) nevertheless holds relatively well (see Table 2).

In this paper we focus on two values for the viscosity which we believe are most relevant (based on the observed accretion rates): low viscosity with $\alpha=10^{-3}$ (arising in zero net flux simulations) and high viscosity with $\alpha=10^{-2}$ (the result of simulations with a weak $\beta=20000$ vertical magnetic field). We use the Pencil Code (Brandenburg 2003) to solve the equations of ideal magnetohydrodynamics, as described in detail in Johansen et al. (2006). For simplicity we ignore vertical stratification of the gas and model a local corotating shearing box with side lengths $1.32 H$, where $H=c_{\mathrm{s}} / \Omega_{\mathrm{K}}$ is the scale height of the gas. Our coordinate frame is oriented in such a way that the $x$-axis points outwards in the radial direction, the $y$-axis points along the main Keplerian flow, while the $z$-axis points vertically out of the disc in the direction of the Keplerian frequency vector $\boldsymbol{\Omega}_{\mathrm{K}}$. The simulation parameters are written in Table 1. The initial condition for the solids is explained in Sect. 3.4.

\subsection{Sweep-up}

Boulders are treated as individual particles, each with a unique position and velocity vector. The boulders feel a drag force from the gas, described in detail in Appendix B, but for simplicity we assume that gas feels no drag from the boulder component. Each particle represents a huge number of actual boulders, hence we refer to them as superparticles. The dust component is treated as a passive scalar: the velocity field is set equal to that of the gas, so that only a continuity equation must be solved, but with additional source and sink terms (due to destruction of boulders and sweep-up) as described below.

The superparticles are given two internal degrees of freedom - the number density of actual boulders inside each particle $\tilde{n}_{i}$ and the average radius $a_{i}$ of the constituent boulders. A superparticle is allowed to change the radius of its boulders, $a_{i}$, by sweeping up dust grains. The dynamical equation for $a_{i}$ is

$\frac{\partial a_{i}}{\partial t}=\frac{\epsilon_{1} \rho_{\mathrm{g}}}{4 \rho_{\bullet}} v_{0 i}$

Here $\epsilon_{1}$ is the dust-to-gas ratio of the dust grains, $\rho_{\mathrm{g}}$ is the density of the gas, $\rho_{\bullet}$ is the material density of the solids, and $v_{0 i}$ is the relative speed between superparticle $i$ and the gas in its grid cell (the dust grains are so coupled to the gas that this is the same as the collision speed between boulders and grains). The density of the dust grains is depleted at the same time according to the evolution equation

$\frac{\partial \epsilon_{1}}{\partial t}=-\tilde{n}_{i} \pi a_{i}^{2} v_{0 i} \epsilon_{1}$ with $\tilde{n}_{i}$ denoting the number density of boulders in the superparticle $i$. This number does not change in a sweep-up process.

\subsection{Collisions between boulders}

We identify all superparticles that reside in the same grid cell as colliding. Collisions between boulders in the superparticles $i$ and $j$ happen at the rate

$\dot{c}_{i j}=\sigma_{i j} \tilde{n}_{i} \tilde{n}_{j} v_{i j}$

where $\sigma_{i j}$ is the collisional cross section and $v_{i j}$ is the collision speed. We assume spherical particles with $\sigma_{i j}=\pi\left(a_{i}+a_{j}\right)^{2}$.

Boulder collisions are assumed to always lead to total destruction of the colliding bodies. The internal number densities of the colliding superparticles $i$ and $j$ change as

$$
\begin{aligned}
\frac{\partial \tilde{n}_{i}}{\partial t} & =-\dot{c}_{i j}, \\
\frac{\partial \tilde{n}_{j}}{\partial t} & =-\dot{c}_{i j},
\end{aligned}
$$

which has to be considered for all combinations of $i$ and $j$ in each grid cell. The total mass that is lost in destructive collisions is subsequently transferred to the dust component. Here the dustto-gas ratio increases as

$$
\rho_{\mathrm{g}} \frac{\partial \epsilon_{1}}{\partial t}=\frac{4}{3} \pi \rho_{\bullet}\left(a_{i}^{3}+a_{j}^{3}\right) \dot{c}_{i j}
$$

One can think of many improvements to these simplified coagulation-fragmentation evolution equations, but we believe that it is enlightening to consider the most simple dynamical equation system that displays coagulation-fragmentation growth. We also compare our results to the advanced models presented in BDH (where a size distribution of solids is considered and where the fragmentation model is much more advanced) and show that our results are in relatively good agreement with the results of $\mathrm{BDH}$.

One may suspect that the sedimentary mid-plane layer could be dense enough to have a gravitational influence on the produced fragments. The gravitational acceleration of a homogeneous, infinitely extended mid-plane layer with density profile $\rho_{\mathrm{p}}(z)=\left[\Sigma_{\mathrm{p}} /\left(\sqrt{2 \pi} H_{\mathrm{p}}\right)\right] \exp \left[-z^{2} /\left(2 H_{\mathrm{p}}^{2}\right)\right]$ is

$g_{z}=-2 \pi G \Sigma_{\mathrm{p}} \operatorname{erf}\left(\frac{z}{\sqrt{2} H_{\mathrm{p}}}\right)$.

This acceleration is 3-4 orders of magnitude less than the vertical gravity from the central star $\left(g_{z}=-\Omega_{\mathrm{K}}^{2} z\right)$. Thus the selfgravity of the sedimentary mid-plane layer can have no influence on the escape rate of fragments and we shall ignore the effect of self-gravity in this paper. 


\subsection{Radial drift}

The accretion process causes the gas pressure in protoplanetary discs to fall with radial distance from the young star. We can write the global radial pressure gradient acceleration as

$-\frac{1}{\rho_{\mathrm{g}}} \frac{\partial P}{\partial r}=-\frac{c_{\mathrm{s}}^{2}}{r} \frac{\partial \ln P}{\partial \ln r}=-\Omega_{\mathrm{K}} c_{\mathrm{s}} \frac{H}{r} \frac{\partial \ln P}{\partial \ln r}$.

Here $H / r$ is the disc aspect ratio and $c_{\mathrm{s}}$ is the sound speed. The balance between Coriolis force and global pressure gradient gives the gas orbital velocity relative to the Keplerian motion as

$\frac{u_{y}^{(\mathrm{subK})}}{c_{\mathrm{s}}}=\frac{1}{2} \frac{H}{r} \frac{\partial \ln P}{\partial \ln r}$.

It is common to define the pressure gradient parameter $\eta$ as (Nakagawa et al. 1986)

$\eta=-\frac{1}{2}\left(\frac{H}{r}\right)^{2} \frac{\partial \ln P}{\partial \ln r}$

giving $u_{y}^{\text {(subK) }}=-\eta v_{\mathrm{K}}$. Here $v_{\mathrm{K}}=\Omega_{\mathrm{K}} r$ is the Keplerian orbital speed.

The boulders do not feel the global pressure gradient and would orbit with the local Keplerian speed in the absence of gas. The head wind of the slower moving gas drains the boulders of angular momentum and imposes a flux of boulders towards smaller $r$. The equilibrium radial drift velocity of the boulders is given by (Weidenschilling 1977; Youdin \& Johansen 2007)

$v_{x}=-\frac{2 \eta v_{\mathrm{K}}}{\Omega_{\mathrm{K}} \tau_{\mathrm{f}}+\left(\Omega_{\mathrm{K}} \tau_{\mathrm{f}}\right)^{-1}}$.

Typical values of $\eta v_{\mathrm{K}}$ lie between $0.02 c_{\mathrm{s}}$ and $0.1 c_{\mathrm{S}}$ (Nakagawa et al. 1986). In this paper we assume that $v_{x}=-0.05 c_{\mathrm{s}}$ for marginally coupled boulders with $\Omega_{\mathrm{K}} \tau_{\mathrm{f}}=1$. As in Johansen et al. (2006) we apply the global pressure gradient force directly on the boulders instead of on the gas. This simplified treatment of radial drift is valid as long as the drag force from the boulders on the gas is ignored.

\subsection{Units and initial condition}

We adopt a dimensionless unit system in our corotating box by setting the sound speed $c_{\mathrm{s}}=1$, Keplerian frequency $\Omega_{\mathrm{K}}=1$ and mid-plane gas density $\rho_{\mathrm{g}}(z=0)=1$ (for computational simplicity we ignore gas stratification, so the gas density is approximately one everywhere in the box). Thus gas and particle velocities are measured in units of the sound speed, while length and particle radius is in units of gas scale heights $H=c_{\mathrm{S}} / \Omega_{\mathrm{K}}=1$. For the magnetic fields we set the vacuum permeability $\mu_{0}=1$. We stress that the role of magnetic fields in our model is to tap into the Keplerian motion and release gravitational energy as turbulent kinetic energy, which is transferred from the gas to the solids by drag forces, but that dust grains and boulders are otherwise unaffected by magnetic fields.

Physical parameters for the different runs are written in Table 1. The initial solids-to-gas ratio of boulders and fragments is set to 0.3 for both species in the two runs where dust fragments are not allowed to diffuse out of the boulder layer (runs A-B), to mimic the density in a sedimentary mid-plane layer ${ }^{3}$, whereas

\footnotetext{
3 It may not be very realistic to have such a high density of small dust grains in a sedimentary mid-plane layer to begin with. Therefore we also ran variations of runs A-B with all the mass initially in the boulder component, but found essentially the same results.
}

the ratio between solids and gas column densities is set to the more canonical 0.01 in the simulations with vertical gravity acting on the boulders (runs C-F). The dynamical equations of coagulation-fragmentation furthermore depend on internal properties of the solids: the radius of the boulders $a_{2}$ and the material density of the solids $\rho_{\mathbf{0}}$. These must be defined in code units. We initially set $a_{2}=10^{-11}$ and $\rho_{\bullet}=10^{11}$, giving an initial Stokes number of unity through $\mathrm{St}_{2}=\Omega_{\mathrm{K}} \tau_{2}=\left(a_{2} / H\right)\left(\rho_{\bullet} / \rho_{\mathrm{g}}\right)=1$.

At $r=5 \mathrm{AU}$ in the minimum mass solar nebula with sound speed $c_{\mathrm{s}}=5 \times 10^{4} \mathrm{~cm} \mathrm{~s}^{-1}$, Keplerian frequency $\Omega_{\mathrm{K}}=1.7 \times$ $10^{-8} \mathrm{~s}^{-1}$ and gas column density $\Sigma_{\mathrm{g}}=150 \mathrm{~g} \mathrm{~cm}^{-2}$ our dimensionless model corresponds to a gas scale height of $H=3 \times$ $10^{12} \mathrm{~cm}$, a mid-plane gas density of $\rho_{\mathrm{g}}(z=0)=2 \times 10^{-11} \mathrm{~g} \mathrm{~cm}^{-3}$, a material density of $\rho_{\bullet}=2 \mathrm{~g} \mathrm{~cm}^{-3}$ and an initial boulder radius of $a_{2}=30 \mathrm{~cm}$.

\section{Results neglecting leaking}

We first treat models where the dust fragments are not allowed to leave the boulder layer (runs A and B in Table 1) to test the validity of the analytical model described in Sect. 2. We mimic the physical conditions in a sedimentary mid-plane layer of boulders by setting the densities of boulders and dust grains artificially high (both components are given a solids-to-gas ratio of 0.3 ). In the following sections, Sects. 5, 6, we treat the more realistic case where boulders lie in a thin layer around the mid-plane and where the dust fragments can leave this layer due to turbulent diffusion. Allowing the dust fragments to escape from the sedimentary mid-plane layer will make prospects to cross the meter barrier by coagulation-fragmentation much more negative than they appear in this section.

In Fig. 3 we show snapshots of the density of fragments (relative to the local gas density) as a function of time. The turbulence has been given 20 orbits to develop before the sweep-up and fragmentation terms are turned on, to avoid the initial condition having any influence on the results. After one orbit (at $t=21 T_{\text {orb }}$ ) fragments have formed in collisions between boulders. The fragments are continuously mixed by the turbulence, and after a few orbits the disc reaches a state where the fragments are very well-mixed with the gas. This state is preferred even though the boulder component is not homogeneous, because the collision time-scale of boulders is much longer than the diffusion time-scale. Considering an overdense region of size $\Delta$, the timescale for fragments to diffuse out of this region is $t_{\text {diff }}=\Delta^{2} / D_{\mathrm{t}}$, where $D_{\mathrm{t}}$ is the diffusion coefficient, while the collisional timescale is $t_{\text {coll }}=m_{2} /\left(\rho_{2} \sigma_{22} v_{22}\right)$. The ratio of the two time-scales is

$$
\frac{t_{\text {diff }}}{t_{\text {coll }}}=\frac{3(\Delta / H)^{2} / \delta}{\Omega_{\mathrm{K}} \tau_{\mathrm{f}}\left(v_{22} / c_{\mathrm{s}}\right)^{-1}\left(\rho_{2} / \rho_{\mathrm{g}}\right)^{-1}} .
$$

Here we have used the parametrisation $D_{\mathrm{t}}=\delta c_{\mathrm{s}}^{2} \Omega_{\mathrm{K}}^{-1}$ and the Epstein friction time $\tau_{\mathrm{f}}=a_{2} \rho_{\bullet} /\left(c_{\mathrm{S}} \rho_{\mathrm{g}}\right)$. Using typical values for run $\mathrm{B}, \Delta / H=0.1, \delta=10^{-2}, \Omega_{\mathrm{K}} \tau_{\mathrm{f}}=1, v_{22} / c_{\mathrm{S}}=0.07$ and $\rho_{2} / \rho_{\mathrm{g}}=1$ in the overdense regions, yields $t_{\text {diff }} / t_{\text {coll }} \approx 0.2$. Thus the fragments have plenty of time to escape the overdense regions before they are swept up by the boulders there, leading to an almost homogeneous spatial distribution of dust fragments at $t=25 T_{\text {orb }}$ in Fig. 3.

In Fig. 4 we show the evolution of the friction time of boulders (first panel), the relative speeds between fragments and boulders $v_{12}$ and between boulders and boulders $v_{22}$ (second panel), the ratio of total fragment mass to total boulder mass $M_{1} / M_{2}$ (third panel), and the analytical expectation for $M_{1} / M_{2}$ (fourth panel) based on Eq. (5). The growth of boulders by 

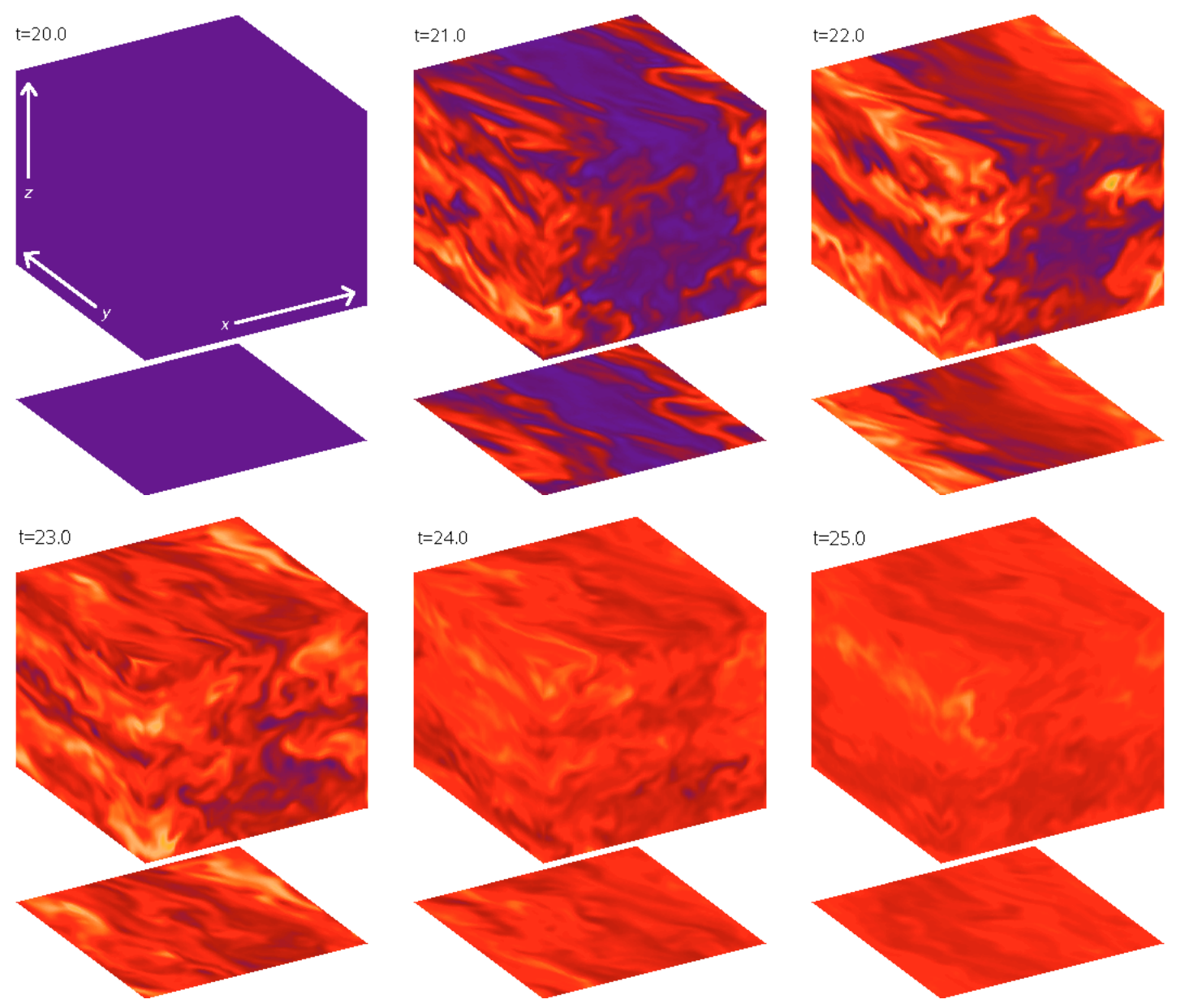

Fig. 3. Time series of the concentration of small dust grains for the run with $\alpha=0.01$ and no vertical gravity for the boulder component (run B). The boxes are oriented with the radial $x$-axis to the right and slightly up, the azimuthal $y$-axis to the left and up, and the vertical $z$-axis directly up. Fragments immediately form in catastrophic collisions between boulders after collisional fragmentation is turned on at $t=20 T_{\text {orb }}$. Even though the boulder component is clumpy, evident from the inhomogeneous initial production of fragments, the dust fragments eventually spread out evenly through the box.

sweep-up is very efficient and allows growth to $\Omega_{\mathrm{K}} \tau_{\mathrm{f}} \gtrsim 10$ in 100-200 orbits, around the same time-scale as the radial drift $^{4}$. At this size (approximately $10 \mathrm{~m}$ ) radial drift is insignificant and the boulders are no longer at risk of being lost to the inner part of the disc. The variation in particle radius at a given time (not shown in Fig. 4) is generally within 10\% of the average value, due to the strong coupling between different regions of the flow by turbulent diffusion.

Average values of collision speeds and boulder growth rates for all the simulations are given in Table 2. The growth rate is almost twice as high in run $\mathrm{B}$ (with $\alpha=10^{-2}$ ) than in run A $\left(\alpha=10^{-3}\right)$ because of the higher collision speeds in the strongly turbulent case.

\subsection{Clumping}

There is a discrepancy of around a factor 2-3 between the analytical expectation of $M_{1} / M_{2}$ in Fig. 4 (panel 4) and the measured value (panel 3). This is likely due to the fact that the boulder layer is not homogeneous because the particles are

\footnotetext{
${ }^{4}$ Note that the Epstein drag law is not valid beyond $\Omega_{\mathrm{K}} \tau_{\mathrm{f}} \approx 7$, see discussion in Appendix B, but since this state can already be considered as having crossed the meter barrier, we have ignored the complications of switching to a Stokes drag law.
}

concentrated in high pressure regions of the gas (Johansen et al. 2006). Fragments are primarily produced in the overdense regions, but they quickly mix with the gas (which is approximately isodense since the turbulence is subsonic). Thus the high density regions must produce more fragments to keep up with the turbulent diffusion, and this increases the overall ratio of fragments to boulders.

One could have thought that local overdensities in the boulder layer would allow for enhanced collisional fragmentation and thus faster growth out of the radial drift regime. This appealing picture nevertheless turns out to be incorrect. To see the effect of clumping we imagine collecting the material from $N$ grid points into one single grid point, or equivalently to take the boulders from a volume $V$ and press them together in the volume $V / N$. The total mass density is $N\left\langle\rho_{1+2}\right\rangle$. The single grid point that contains boulders must fulfil Eq. (5) in order to be in equilibrium. This leads to the equation system

$N \rho_{1}+\rho_{2}=N\left\langle\rho_{1+2}\right\rangle$,
$\rho_{1}-4 \zeta \rho_{2}=0$,

where we define $\zeta \equiv v_{22} / v_{12}$ and assume that the diffusion timescale is much shorter than the collisional time-scale, so that $\rho_{1}$ 
1

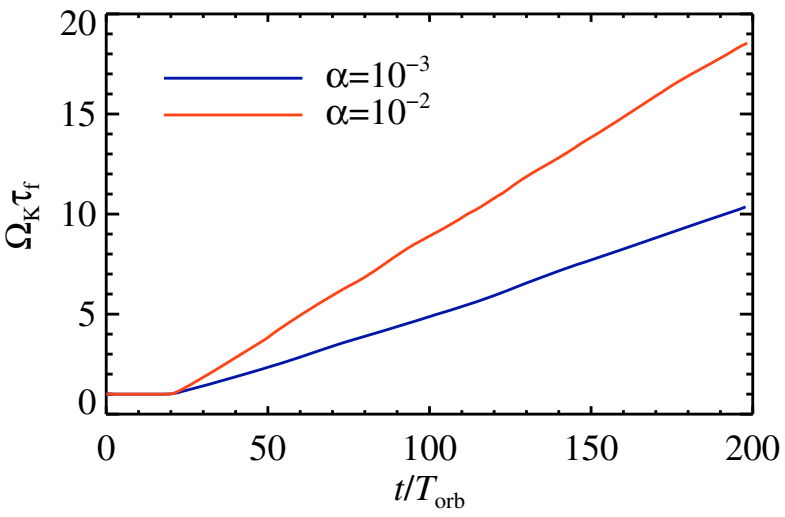

3

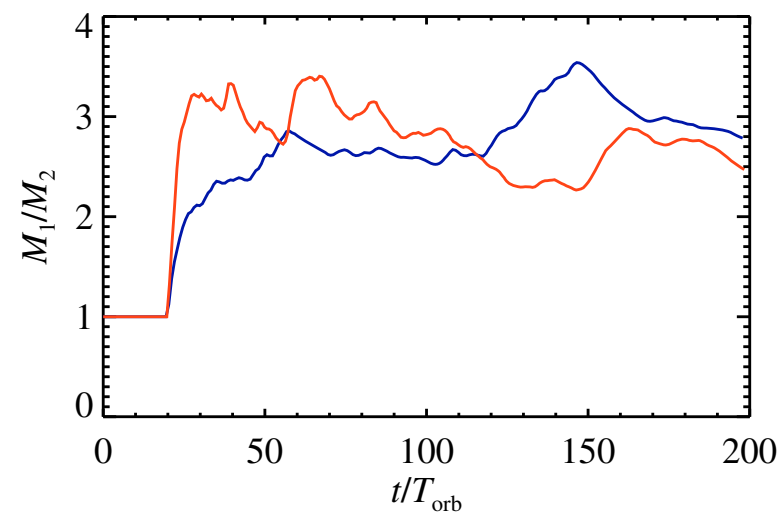

\section{2}

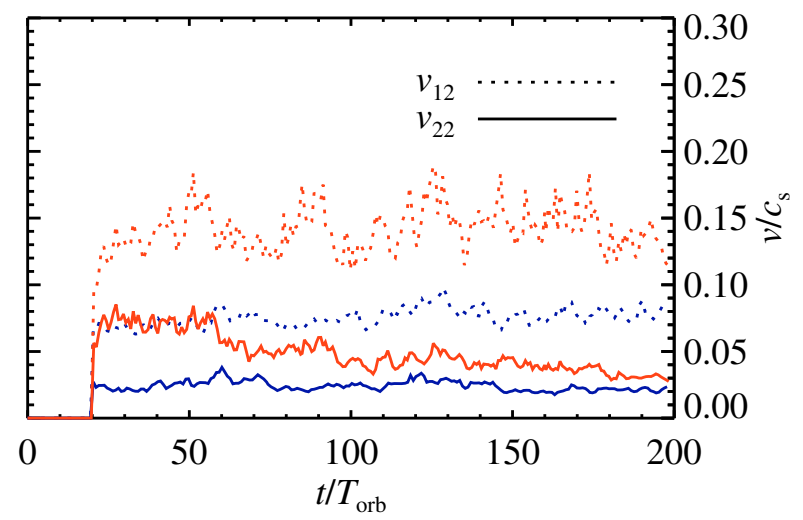

4

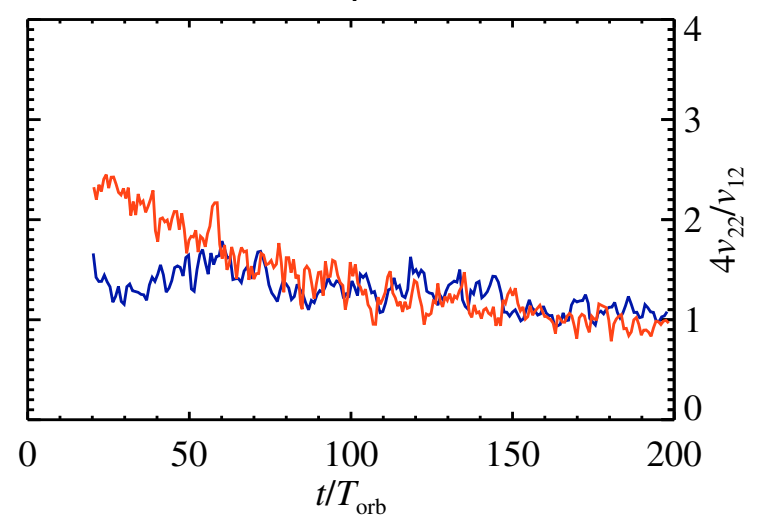

Fig. 4. Growth and fragmentation of boulders as a function of time $t$, measured in orbits, for two different strengths of the turbulence. The first panel shows the friction time of the boulders: growth from the initial $\Omega_{\mathrm{K}} \tau_{\mathrm{f}}=1$ to beyond $\Omega_{\mathrm{K}} \tau_{\mathrm{f}}=10$ occurs readily for both weak and strong turbulence, although the stronger turbulence helps growth by producing more fragments. The second panel shows the relative speeds between boulders and fragments $\left(v_{12}\right)$ and between boulders and boulders $\left(v_{22}\right)$. The boulder collision speed decreases with time as the particles grow and decouple from the gas, whereas the relative speed between grains and boulders is set primarily by the turbulent motion of the dust grains and thus stays approximately constant. The total mass of the fragments, $M_{1}$, reaches $2-3$ times the mass of boulders, $M_{2}$, in the equilibrium state (panel 3), somewhat higher than the analytical expectation (panel 4) based on Eq. (5), but that is likely due to the fact that the boulder density is not homogeneous: fragments are created where the boulder density is high, but quickly escape these regions by turbulent diffusion, leading to an increase in the total amount of dust.

will be constant over all grid cells. The solution to the algebraic equation system is

$$
\begin{aligned}
& \rho_{1}=\frac{N}{N+1 /(4 \zeta)}\left\langle\rho_{1+2}\right\rangle, \\
& \rho_{2}=\frac{N}{4 \zeta N+1}\left\langle\rho_{1+2}\right\rangle .
\end{aligned}
$$

For $N=1$, corresponding to no clumping, we recover the usual $\rho_{1}=(4 / 5)\left\langle\rho_{1+2}\right\rangle$ and $\rho_{2}=(1 / 5)\left\langle\rho_{1+2}\right\rangle$ for $\zeta=1$. For $N \rightarrow \infty$ the expressions tend towards $\rho_{1}=\left\langle\rho_{1+2}\right\rangle$ and $\rho_{2}=[1 /(4 \zeta)]\left\langle\rho_{1+2}\right\rangle$. Thus clumping has little or no effect on the equilibrium density of dust fragments, which is the crucial parameter that determines radius growth. The overdense regions must produce enough dust not only to feed its own zone, but also to fill up the regions that contain no boulders, as any gradients in the dust density will be quickly (instantaneously actually, in the simplified model presented in Eqs. (30), (31)) evened out by diffusion. Clumping leads at most to a $25 \%$ increase in radius growth, but at the cost of reducing the total number of boulders proportionally to the degree of clumping. We investigate the role of turbulent diffusion further in Sect. 5.

\subsection{Recycling time-scale}

Even though a balance between sweep-up and collisional fragmentation arises, so that the number density of fragments $n_{1}$ stays approximately constant in time, there is a significant flux of dust grains through the boulder component. The evolution equation for $n_{1}$ (Eq. (1)) consists of two terms that balance out at equilibrium. One can rewrite the evolution equation in terms of the time-scale for sweep-up $t_{\text {sweep }}$ and the time-scale for replenishment of dust fragments by boulder collisions $t_{\text {replenish }}$,

$\frac{1}{\rho_{1}} \frac{\partial \rho_{1}}{\partial t}=-\frac{1}{t_{\text {sweep }}}+\frac{1}{t_{\text {replenish }}}$

At equilibrium the sweep-up and replenishment time-scales are equal. The sweep-up time-scale is

$t_{\text {sweep }}=\frac{1}{n_{2} \sigma_{12} v_{12}}$

Inserting the equilibrium solution $\rho_{1} / \rho_{2}=4 v_{22} / v_{12}$ from Eq. (5) gives

$t_{\text {sweep }}=\frac{1+4 v_{22} / v_{12}}{v_{12}} \frac{a_{2} \rho_{\bullet}}{3 \rho_{1+2}}$ 
Table 2. Results. The collision speeds $v_{12}$ and $v_{22}$ are averaged over orbits 20-30, while the column density ratio $\Sigma_{1} / \Sigma_{2}$ and boulder radius growth rate $\dot{a}_{2}$ (given here in Stokes number per orbit) are averaged over the last 10 orbits in the simulation. The recycling time-scale $t_{\text {recyc }}$ is calculated from Eqs. (37) and (51). Measurements marked with * had not yet saturated at the end of the simulation.

\begin{tabular}{crccccccr}
\hline \hline Run & Res & Leaking & $\alpha$ & \multicolumn{1}{c}{$v_{12}$} & $v_{22}$ & $\Sigma_{1} / \Sigma_{2}$ & $\dot{a}_{2}$ & $t_{\text {recyc }}$ \\
\hline A & $64^{3}$ & No & $10^{-3}$ & 0.067 & 0.023 & 2.82 & 0.057 & 3.1 \\
B & $64^{3}$ & No & $10^{-2}$ & 0.12 & 0.071 & 2.54 & 0.089 & 2.5 \\
C & $64^{3}$ & Yes & $10^{-3}$ & 0.066 & 0.014 & $94.0^{*}$ & 0.0036 & 803.5 \\
D & $64^{3}$ & Yes & $10^{-2}$ & 0.12 & 0.054 & 57.6 & 0.0064 & 306.4 \\
E & $128^{3}$ & Yes & $10^{-3}$ & 0.063 & 0.010 & $58.0^{*}$ & 0.0029 & 640.5 \\
$\mathrm{~F}$ & $128^{3}$ & Yes & $10^{-2}$ & 0.16 & 0.075 & $40.0^{*}$ & 0.0068 & 238.6 \\
\hline
\end{tabular}

when assuming spherical grains with material density $\rho_{\bullet}$. One can simplify the equation further by inserting the Epstein regime friction time from Eq. (12), yielding

$\frac{t_{\text {sweep }}}{\tau_{\mathrm{f}}}=\frac{1+4 v_{22} / v_{12}}{3}\left(\frac{\rho_{1+2}}{\rho_{\mathrm{g}}}\right)^{-1}\left(\frac{v_{12}}{c_{\mathrm{s}}}\right)^{-1}$.

This is the time-scale upon which the boulders would empty the grain component in the absence of collisional fragmentation. In coagulation-fragmentation equilibrium, collisional fragmentation produces dust grains at the same rate as they are swept up. But the sweep-up time-scale can also be associated with a characteristic recycling time-scale. On average dust fragments spend the time $t_{\text {sweep }}$ in the grain component, before they are incorporated into a boulder. We have calculated the recycling time-scale for runs A and B, based on Eq. (37), in Table 2. The grains have a recycling time-scale of around three orbits in the two runs where dust fragments cannot leave the boulder layer. Thus the grains have been through approximately 100 agglomerationdestruction cycles during the course of runs A and B. We return to the recycling time-scale in models where dust fragments are allowed to diffuse out of the mid-plane layer in Sect. 6.1.

\section{Diffusion}

In this section we generalise the analytical model of Sect. 2 to 1 -D. Adding a $z$-direction to the problem and exposing the dust particles to turbulent diffusion and vertical gravity yields the following equation system for $\rho_{1}=m_{1} n_{1}$ and $\rho_{2}=m_{2} n_{2}$ :

$$
\begin{aligned}
\frac{\partial \rho_{1}}{\partial t}= & -\rho_{1} \rho_{2} \sigma_{12} v_{12} \frac{1}{m_{2}}+\rho_{2}^{2} \sigma_{22} v_{22} \frac{1}{m_{2}} \\
& -\frac{\partial\left(w_{1} \rho_{1}\right)}{\partial z}+D_{1} \frac{\partial}{\partial z}\left[\rho_{\mathrm{g}} \frac{\partial\left(\rho_{1} / \rho_{\mathrm{g}}\right)}{\partial z}\right], \\
\frac{\partial \rho_{2}}{\partial t}= & +\rho_{1} \rho_{2} \sigma_{12} v_{12} \frac{1}{m_{2}}-\rho_{2}^{2} \sigma_{22} v_{22} \frac{1}{m_{2}} \\
& -\frac{\partial\left(w_{2} \rho_{2}\right)}{\partial z}+D_{2} \frac{\partial}{\partial z}\left[\rho_{\mathrm{g}} \frac{\partial\left(\rho_{2} / \rho_{\mathrm{g}}\right)}{\partial z}\right] .
\end{aligned}
$$

Here $D_{1}$ and $D_{2}$ is the turbulent diffusion coefficient of grains and boulders, respectively, and $w_{1}$ and $w_{2}$ are the vertical velocities, assumed to be in equilibrium between gravity and drag force with

$w_{i}=-\frac{z}{t_{\mathrm{sett}}} \approx-\frac{\Omega_{\mathrm{K}}^{2} \tau_{i}}{1+\Omega_{\mathrm{K}}^{2} \tau_{i}^{2}} z$.

This approximate expression recovers the terminal velocity of the grains in the small friction time regime, $w_{i}=-\tau_{i} \Omega_{\mathrm{K}}^{2} z$, and the settling time of oscillating particles in the large friction time regime, $t_{\mathrm{sett}}=1 / \tau_{i}$ (Youdin \& Lithwick 2007). The diffusion coefficient $D_{i}$ depends on the friction time of the particles as (see Carballido et al. 2006; Youdin \& Lithwick 2007)

$D_{i}=\frac{D_{0}}{1+\Omega_{\mathrm{K}}^{2} \tau_{i}^{2}}$

where $D_{0}$ is the diffusion coefficient of a passive scalar. We assume that the turbulent mixing is independent of the height over the mid-plane, i.e. that $D_{0}$ is a constant. Ignoring the fragmentation and sweep-up terms of Eqs. (38) and (39) allows for a simple equilibrium between diffusion and sedimentation,

$\rho_{i}(z)=\frac{\Sigma_{i}}{\sqrt{2 \pi} H_{i}} \exp \left[-z^{2} /\left(2 H_{i}^{2}\right)\right]$,

where the scale-height $H_{i}$ obeys the relation

$\frac{1}{H_{i}^{2}}=\frac{1}{H^{2}}+\frac{\Omega_{\mathrm{K}}^{2} \tau_{i}}{D_{0}}$

and $\Sigma_{i}$ is the total column density of solids of type $i$. Here the denominators of Eqs. (40) and (41) have cancelled, making the short friction time scale height expression (Eq. (43)) valid for all particle sizes. However, the equilibrium solution in Eqs. (42), (43) is formally only valid when the friction time $\tau_{i}$ is assumed to be constant with height over the mid-plane, an assumption that breaks down when considering the dust distribution over several scale heights (Dullemond \& Dominik 2004).

Integrating Eqs. (38) and (39) over the entire $z$-space yields dynamical equations for the column densities $\Sigma_{1}$ and $\Sigma_{2}$ instead,

$\frac{\partial \Sigma_{1}}{\partial t}=\int_{-\infty}^{\infty}\left(-\rho_{1} \rho_{2} \sigma_{12} v_{12} \frac{1}{m_{2}}+\rho_{2}^{2} \sigma_{22} v_{22} \frac{1}{m_{2}}\right) \mathrm{d} z$,

$\frac{\partial \Sigma_{2}}{\partial t}=\int_{-\infty}^{\infty}\left(+\rho_{1} \rho_{2} \sigma_{12} v_{12} \frac{1}{m_{2}}-\rho_{2}^{2} \sigma_{22} v_{22} \frac{1}{m_{2}}\right) \mathrm{d} z$,

where the derivative terms have vanished because the column density of solids cannot be changed by sedimentation and vertical diffusion. Inserting the sedimentation-diffusion equilibrium solution from Eq. (42) into Eqs. (44) and (45) and searching for $\Sigma_{1} / \Sigma_{2}$ that gives $\dot{\Sigma}_{1}=\dot{\Sigma}_{2}=0$ yields

$\frac{\Sigma_{1}}{\Sigma_{2}}=\frac{\sigma_{22}}{\sigma_{12}} \frac{v_{22}}{v_{12}} \frac{\sqrt{1+H_{1}^{2} / H_{2}^{2}}}{\sqrt{2}}$

as an extension to Eq. (5). If $\tau_{1}=\tau_{2}$, then $H_{1}=H_{2}$ according to Eq. (43), and $\Sigma_{1} / \Sigma_{2}=\left(\sigma_{22} / \sigma_{12}\right)\left(v_{22} / v_{12}\right)$, equivalent to the 0-D case. Combining Eqs. (42), (43) and (46) and inserting $\rho_{1}(z=0)$ in Eq. (4) yields the radius growth of boulders in the mid-plane as

$\dot{a}_{2}=\frac{\Sigma_{1+2} /\left(\sqrt{2 \pi} H_{1}\right)}{\rho_{\bullet}} \frac{v_{22}}{\left[2 /\left(1+H_{1}^{2} / H_{2}^{2}\right)\right]^{1 / 2}+4 v_{22} / v_{12}}$,

the 1-D generalisation of Eq. (6). The growth rate of the boulders is approximately inversely proportional to $H_{1}$ (note that the $H_{1}^{2} / H_{2}^{2}$ term in the denominator of Eq. (47) has little influence on the growth rate for $H_{1} \gg H_{2}$ ). Thus going from $H_{1}=H_{2}$ to, say, $H_{1}=10 H_{2}$, a reasonable value for small fragments, decreases the growth rate by coagulation-fragmentation by a dramatic order of magnitude.

The validity of the Gaussian solution (Eq. (42)) can be quantified by comparing the relevant time-scales of Eqs. (38) 

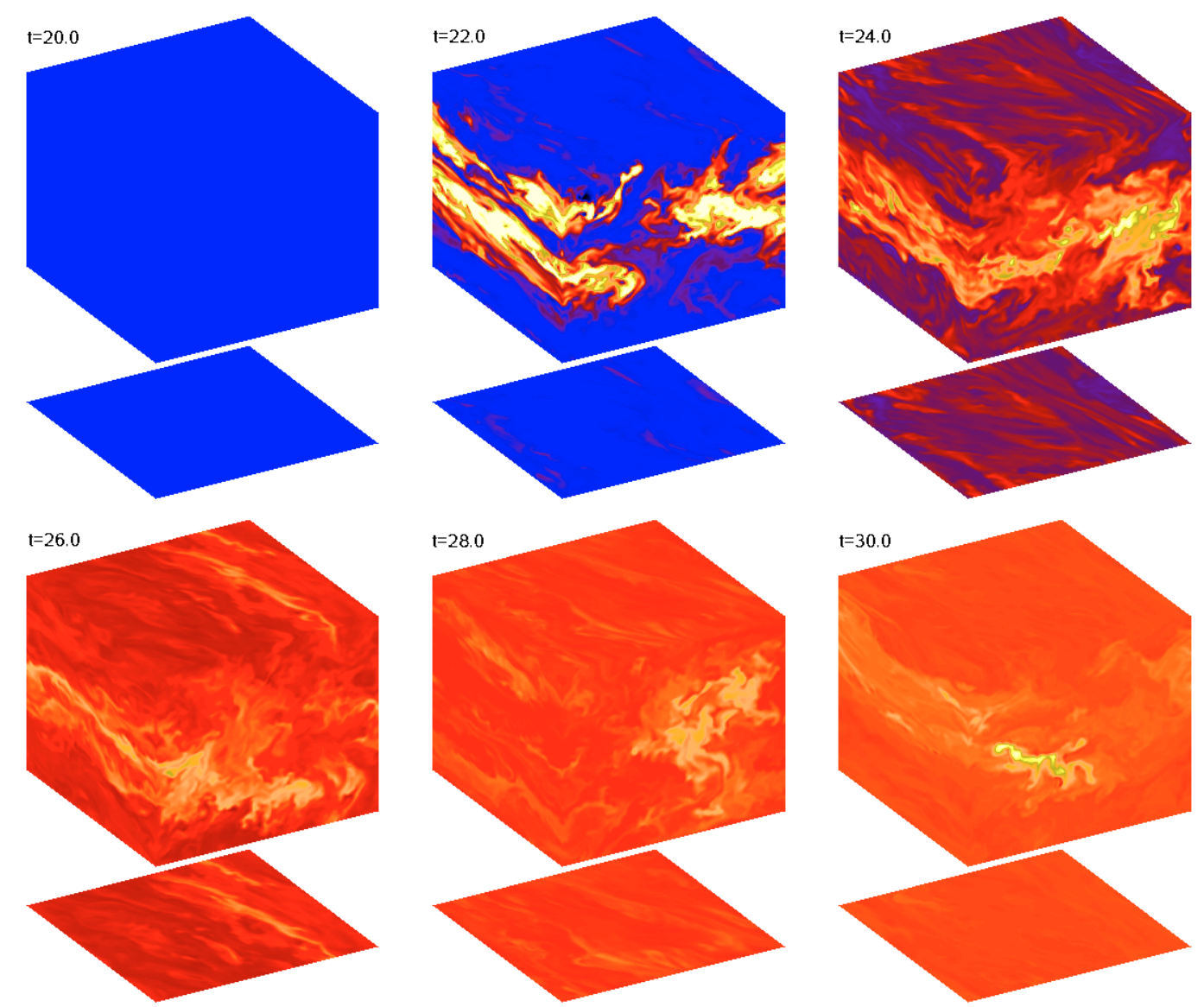

Fig. 5. Time series of the concentration of small dust grains for a run with $\alpha=0.01$ where the boulders lie in a thin layer around the mid-plane of the disc (run F). Fragments form when boulders collide in the mid-plane, but the produced dust grains escape from the mid-plane due to turbulent diffusion, eventually spreading evenly over the box.

and (39) - the time-scale for fragments to diffuse out of the mid-plane layer, $t_{\text {diff }}=H_{2}^{2} / D_{\mathrm{t}}$, and the collisional time-scale, $t_{\text {coll }}=m_{2} /\left(\rho_{2} \sigma_{22} v_{22}\right)$. The Gaussian solution is valid when $t_{\text {diff }} \ll t_{\text {coll }}$, giving

$\Omega_{\mathrm{K}}^{2} \tau_{2}^{2} \gg \frac{\rho_{2}}{\rho_{\mathrm{g}}} \frac{v_{22}}{c_{\mathrm{s}}}$,

where we used $\tau_{2}=\left(a_{2} \rho_{\bullet}\right) /\left(c_{\mathrm{s}} \rho_{\mathrm{g}}\right)$ to translate the sedimentation time-scale into an Epstein friction time. Further using the sedimentation-diffusion equilibrium expression for the midplane density $\rho_{2} / \rho_{\mathrm{g}}=\epsilon_{0} \sqrt{\Omega_{\mathrm{K}} \tau_{\mathrm{f}} / \delta}$, where $\epsilon_{0}$ is the global solidsto-gas ratio of the boulder component (see e.g. Johansen et al. 2006), yields

$\Omega_{\mathrm{K}} \tau_{\mathrm{f}} \gg \delta^{-1 / 3}\left(\epsilon_{0} \frac{v_{22}}{c_{\mathrm{s}}}\right)^{2 / 3}$

with fully independent parameters (both the collision speed $v_{22}$ and the diffusion coefficient $\delta$ of course depend on the strength of the turbulence, so these two must be chosen consistently). For $\delta=10^{-3}, \epsilon_{0}=0.01$ and $v_{22} / c_{\mathrm{s}}=0.02$ the criterion for the validity of the Gaussian solution is $\Omega_{\mathrm{K}} \tau_{2} \gg 0.03$, in accordance with our modelling of component 2 as boulders with $\Omega_{\mathrm{K}} \tau_{\mathrm{f}} \gtrsim 1$.

The simple model presented in Sect. 4.1 for the effect of clumping on coagulation-fragmentation also gives a new perspective on the effect of sedimentation (Eqs. (46) and (47)). Decreasing the scale height of boulders from $H_{2}=H_{1}$ to $H_{2} \ll H_{1}$ leads to a steep rise in $\Sigma_{1} / \Sigma_{2}$ (Eq. (46)), but only to a mild increase in radius growth (Eq. (47)). Thus any nonhomogeneity of the boulder layer (be it due to concentrations in transient gas high pressure regions or due to sedimentation) has little effect on the growth rate of the boulders, but may reduce the bulk density of boulders drastically. Taking instead the scale height of fragments $H_{1}$ and increasing it from $H_{1}=H_{2}$ to $H_{1} \gg H_{2}$, a transition that is similar to the one occurring from models A-B to models C-F, leads to a sharp decrease in both growth rate and column density of the boulder component.

\section{Results including leaking}

Having found in the preceding section an approximate analytical solution for the coagulation-fragmentation equilibrium in the case where dust fragments are free to leave the sedimentary boulder layer, we now return to the results of numerical simulations. In runs C-F we set the solids-to-gas ratio of boulders and fragments to the more canonical value 0.01 and expose the boulders to vertical gravity. We give the boulders time to settle to the mid-plane from $t=10 T_{\text {orb }}$ to $t=20 T_{\text {orb }}$, so that an equilibrium sedimentary layer has formed when sweep-up and collisional fragmentation are turned on at $t=20 T_{\text {orb }}$.

In Figs. 5 and 6 we show the time evolution of fragments and boulders in models where the boulders have sedimented out of the gas to establish a thin layer around the mid-plane of the box. Fragments form in catastrophic collisions between boulders in the mid-plane, but are quickly carried away from the midplane layer by the turbulent gas and are eventually well-mixed throughout the box (Fig. 5). Figure 6 shows, for two different numerical resolutions, that the boulders grow by a factor ten slower than in the case where dust fragments were not allowed 
1

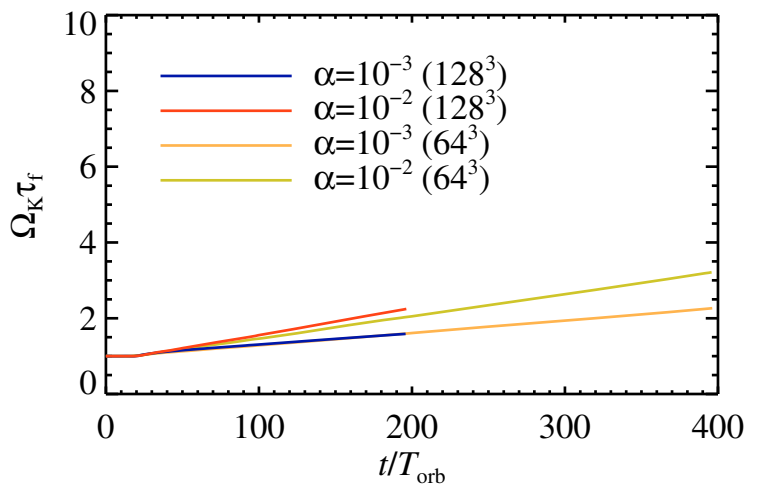

3

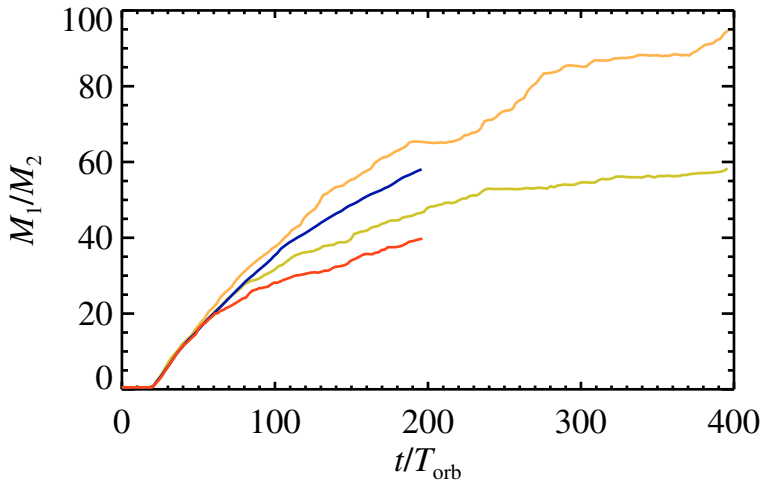

2

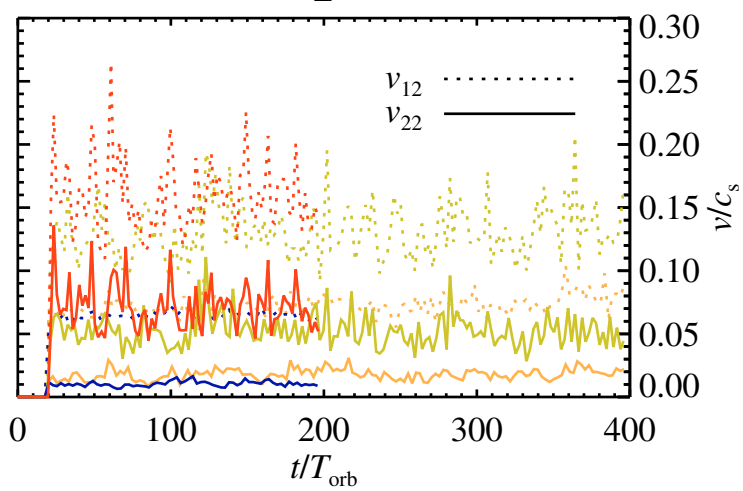

4

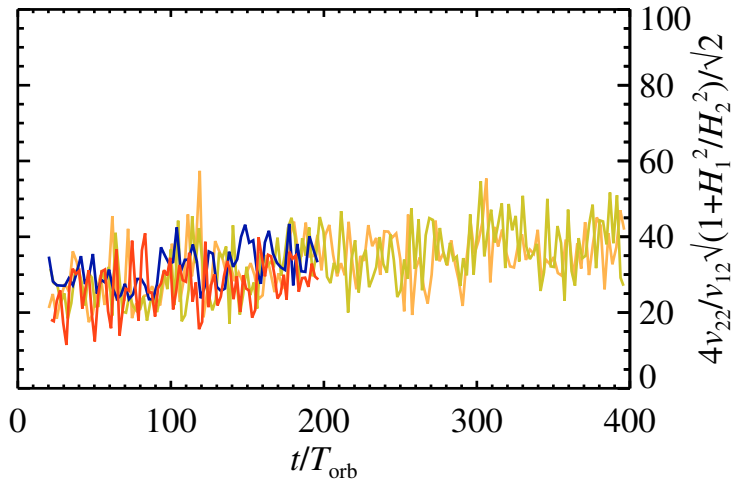

Fig. 6. Growth and fragmentation of boulders in models where the dust fragments are allowed to diffuse out of the boulder layer. The radius of the boulders increases much slower than in Fig. 4, since fragments created in catastrophic collisions easily escape from the mid-plane layer and spread over the entire vertical extent of the disc. The ratio of fragments to boulders (panel 3) approximately matches the value expected from analytical derivations (panel 4) based on the different equilibrium scale height of boulders and fragments. Due to the slow growth boulders will have drifted into the inner disc to evaporate there before the meter barrier would eventually be crossed after a few thousand orbits.

to leave the boulder layer (Fig. 4). Also the mass of fragments is huge because the catastrophic collisions have to keep up with the turbulent transport of fragments away from the mid-plane. The fourth panel of Fig. 6 shows the analytical expectation value for $M_{1} / M_{2}$, following Eq. (46), by setting the expected scale height of fragments, $H_{1}$, equal to the gas scale height and using the collision speeds from the second panel. There is slightly more mass in fragments than expected, which may, as in the case where dust fragments were not allowed to leave the boulder layer shown in Fig. 4, be explained by the clumpy structure of the boulder layer (see Sect. 4.1).

Turbulent transport reduces the column density of boulders to approximately $1-2 \%$ of the total column density of the solids. The growth rate of the Stokes number of the boulders is around $0.003 \ldots 0.007$ per orbit, with the higher values appearing in the strongly turbulent $\alpha=10^{-2}$ runs. Under all circumstances this is far too low to compete with radial drift which occurs on a time-scale of a few tens of orbits. Even if the radial drift timescale is ignored it would take around 1000 orbits to grow to $\mathrm{St}=10$. The extremely low column density of the boulders corresponds to around 1 boulder per $(10 \mathrm{~km})^{2}$. It is surprising that so few boulders can populate the entire vertical extent of the disc with small dust grains. Eisner et al. (2006) recently modelled submicron-sized dust grains in the inner part of the transition disc TW Hya and found that the lifetimes of these grains against radiation pressure is much shorter than the age of the system. One can speculate that the source of these small grains could be boulders (drifting through the inner disc or permanently situated there) creating observable numbers of fragments as they collide.

The minimum and maximum bulk density of fragments in the gas is shown in Fig. 7. After collisional fragmentation is turned on at $t=20 T_{\text {orb }}$, there is a sharp increase in the maximum concentration of fragments, but turbulent mixing eventually leads to a state where the minimum and maximum concentrations are within $3 \%$ of each other for $\alpha=10^{-2}$ and within $6 \%$ of each other for $\alpha=10^{-3}$. It may be surprising that the difference is so small, but it is again because the collisional time-scale is much longer than the diffusion time-scale. This also explains why the $\alpha=10^{-2}$ has less difference between minimum and maximum concentrations than $\alpha=10^{-3}-$ the stronger mixing in the highly turbulent case evens out concentration differences more effectively. We note that although the average solids-togas ratio in the disc is assumed to be $\epsilon_{0}=0.02$, the fragments reach an average bulk density of $\epsilon_{0}=0.029$. This is an artificial effect of the limited vertical extent of the box: boulders have sedimented out of the regions outside of the box, but fragments are not allowed to escape back out the box, hence the average density of fragments is kept artificially high.

\subsection{Recycling time-scale revisited}

We introduced the recycling time-scale in Sect. 4.2. With diffusion of dust fragments from the boulder layer suppressed, the dust grains would be free for only around three orbits before 


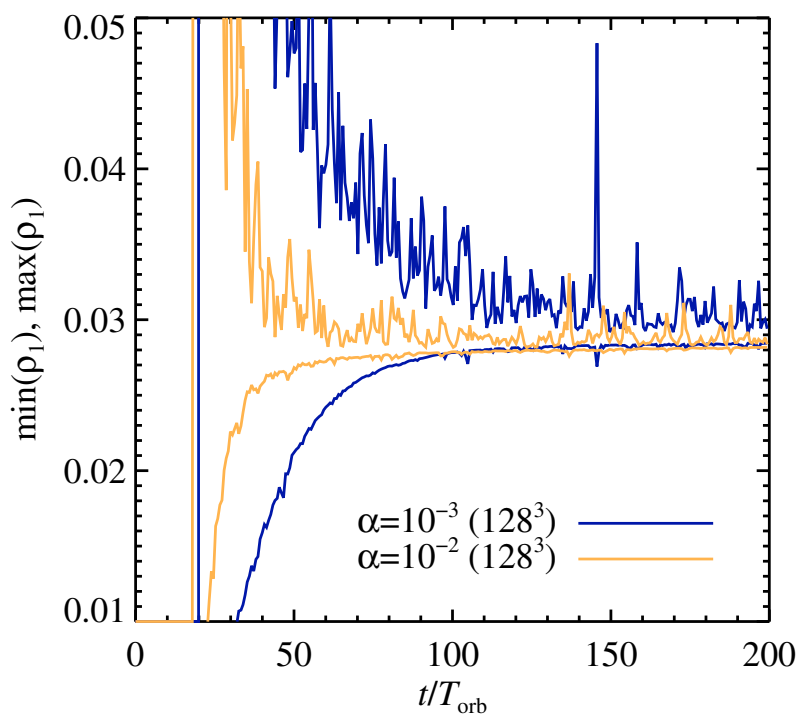

Fig. 7. The minimum and maximum bulk density of fragments, $\rho_{1}$, in the box as a function of time for runs $\mathrm{E}$ and $\mathrm{F}$. Collisional fragmentation is turned on at $t=20 T_{\text {orb }}$, followed by an initial peak in the maximum density of fragments. Turbulent mixing nevertheless leads to an almost homogeneous state with only very little difference between minimum and maximum concentrations of fragments. The case $\alpha=10^{-2}$ has less difference between minimum and maximum concentrations than for $\alpha=10^{-3}$ due to stronger mixing in the first case.

being incorporated into a boulder. We now derive a similar expression for the recycling time-scale, valid in the case where dust fragments are allowed to diffuse out of the thin mid-plane layer where they originate in collisions between boulders.

Assuming that the density dependence on height over the mid-plane is Gaussian for both species (Eq. (42)) we can evaluate the integral in Eq. (44) analytically and obtain the recycling time-scale

$t_{\text {recyc }}=\frac{\sqrt{2 \pi} \sqrt{H_{1}^{2}+H_{2}^{2}}}{\Sigma_{2}} \frac{m_{2}}{\sigma_{12} v_{12}}$.

Notice that the recycling time-scale for $H_{1}=H_{2}$ is $\sqrt{2}$ times longer than in the case where dust fragments and boulders were not allowed to separate vertically, because, although the densities in the mid-plane are exactly as in the 0-D case, the vertically averaged collision rate is lower, which leads to a somewhat longer recycling time-scale.

Inserting the coagulation-fragmentation equilibrium from Eq. (46) yields the recycling time-scale in units of the friction time as

$$
\begin{aligned}
\frac{t_{\text {recyc }}}{\tau_{\mathrm{f}}}= & \frac{1+4\left(v_{22} / v_{12}\right)\left(\sqrt{1+H_{1}^{2} / H_{2}^{2}} / \sqrt{2}\right)}{3} \\
& \times\left[\frac{v_{12}}{c_{\mathrm{s}}}\right]^{-1}\left[\frac{\Sigma_{1+2} /\left(\sqrt{2 \pi} \sqrt{H_{1}^{2}+H_{2}^{2}}\right)}{\rho_{\mathrm{g}}}\right]^{-1},
\end{aligned}
$$

equivalent to Eq. (37). Using $\Sigma_{1+2}=0.02 \Sigma_{\mathrm{g}}=0.02 \sqrt{2 \pi}$ in units where the mid-plane gas density is unity, together with $H_{1} / H_{\mathrm{g}}=1$ and the diffusion-sedimentation equilibrium $\left.H_{2} / H_{\mathrm{g}}=\sqrt{\delta /\left(\Omega_{\mathrm{K}} \tau_{\mathrm{f}}\right.}\right)$, we have calculated the recycling timescale based on Eq. (51) in Table 2. Allowing dust fragments to leak out of the mid-plane layer leads to a dramatic increase by two orders of magnitude in the recycling time-scale, which is now 200-300 orbits for the $\alpha=10^{-2}$ models and approaching a thousand orbits for the $\alpha=10^{-3}$ runs. Not only does the vertical diffusion decrease the amount of boulders in the mid-plane by approximately an order of magnitude, leading to a much longer sweep-up time-scale, but the total amount of dust grains is also higher, so that it takes a much longer time for the average dust grain to encounter a boulder.

\section{Summary and discussion}

We have proposed a simple two-component model for the growth and collisional destruction of boulders in protoplanetary discs. Fragments produced in catastrophic collisions between boulders are swept up by other boulders, leading to a continuous growth towards larger bodies. An analytical equilibrium solution to the dynamical equations predicts that the boulder radius can grow as quickly as a few mm per year. The promise of this method to provide growth rates that can compete with the radial drift is nevertheless compromised by the inclusion of turbulent transport of fragments out of the boulder layer. The sedimentary mid-plane layer loses the produced fragments to the boulder-free parts of the disc, eventually grinding down the boulder component to insignificant masses and reducing the growth rate drastically. Thus what initially seemed to have the potential to provide an efficient growth phase of boulders turned into something like a worst-case scenario with the inclusion of turbulent transport.

One can think of ways by which boulders may still penetrate the meter barrier by sweep-up of small fragments:

$$
\begin{aligned}
& \text { - fragments are larger; } \\
& \text { - boulder collisions do not lead to destruction; } \\
& \text { - protoplanetary discs are less turbulent than assumed; } \\
& \text { - turbulence is confined to the mid-plane; } \\
& \text { - radial drift is weaker in nature than thought. }
\end{aligned}
$$

If fragments are large enough that they do not couple instantaneously (compared to an orbital time-scale) to the gas, then the turbulent transport away from the sedimentary mid-plane layer is slowed down. Having such large fragments would in principle put coagulation-fragmentation growth back on track. BDH presented models with a much more advanced collisional fragmentation model, where the results of catastrophic collisions are distributed with a power law, but this still did not lead to a breakthrough of the meter barrier. If, on the other hand, boulders do not fragment at all, then the whole concept of coagulationfragmentation growth breaks down, and the stage is left to coagulation of equal-sized boulders and/or self-gravity in the boulder component (Johansen et al. 2007).

Turbulence plays an interesting double role in the coagulation-fragmentation process. The relative speed between the boulders, which leads to their destruction and the continuous replenishment of the grains, is induced by the marginal coupling of the boulders to the turbulent gas motion. On the other hand turbulent diffusion drains the mid-plane layer of fragments and reduces the growth rate of the boulders. This is in some opposition to the role that is normally attributed to turbulence in the coagulation process. Here a dense mid-plane layer and reduced collisional fragmentation are desired, and turbulence counteracts both. Coagulation-fragmentation growth, on the other hand, benefits directly from stronger turbulence and higher collision speeds (although the sweep-up efficiency can be put into question when the relative speed between dust grains and boulders increases beyond a few tens of meters per second, see discussions 
in Wurm et al. 2001, 2005), while it is indifferent to sedimentation and clumping of the boulder layer.

The presented simulations all have a simplified spacefilling turbulence of magnetic origin. If turbulence would instead be confined to the mid-plane of the disc, as assumed in Weidenschilling (1997), then the transport of grains away from the mid-plane can be reduced, while at the same time the collision speeds can be kept high. The Kelvin-Helmholtz and streaming instabilities associated with the sedimentation of solids are nevertheless not Keplerian shear instabilities (Youdin \& Goodman 2005; Youdin \& Johansen 2007) and thus cannot explain the observed accretion rates of young stars. If, on the other hand, there is a region around the mid-plane where the magnetic field does not couple well enough to the gas to have magnetorotational instability (Gammie 1996; Oishi et al. 2007), then one could have lower turbulence in the mid-plane and much less loss of fragments to the boulder-free parts of the disc, but as discussed above, a net decrease of turbulence in the mid-plane has a negative impact on the coagulation-fragmentation growth. Dust grains are a major catalyst for recombination of ionised species (Sano et al. 2000). This leads to an interesting coupling between dust and turbulence whereby dusty regions would have weaker turbulent motion, and thus less diffusion, than dust-free regions. We plan to include such effects in a future model.

Collisional fragmentation is actually not the real problem for planetesimal formation, given that fragments are readily incorporated in the few lucky boulders that avoid catastrophic collisions. The problem arises because the time-scale of growth by coagulation-fragmentation is so long that all material will have been flushed through the disc long before being able to grow large enough to decouple from the gas. Radial drift in the minimum mass solar nebula reaches $5 \%$ of the local sound speed (Weidenschilling 1977), yielding a drift time-scale of a few tens of orbits, much too short for any significant radius growth.

There is mounting observational evidence that radial drift may not be as great in actual discs as predicted from theoretical arguments. Rettig et al. (2006) measured the abundance of gas and dust in discs of millions of years in age and were able to explain the inclination dependence of the dust-to-gas ratio from a combination of grain growth and sedimentation, still within the framework of the minimum mass solar nebula with a solids-togas ratio of 0.01 . In the models of drift and coagulation presented in $\mathrm{BDH}$, on the other hand, the outer disc is cleared of dust in a few hundred thousand years. The observed presence of $\mathrm{cm}$-sized solids in the outer parts of protoplanetary discs (Wilner et al. 2000; Testi et al. 2003; Rodmann et al. 2006; Lommen et al. 2007 ) is at best marginally consistent with theoretical life-times of such grains due to radial drift (Brauer et al. 2007). Maybe the most promising way to stop radial drift is to have radial pressure bumps in the disc (Whipple 1972). These bumps can arise from first principles in 3-D simulations of the dynamics of protoplanetary discs, e.g. spiral arms of self-gravitating discs (Rice et al. 2004) or long-lived high pressure regions in magnetorotational turbulence (Fromang \& Nelson 2005; Johansen et al. 2006). Any pressure bump must compete with the global pressure gradient and produce a net zero or positive gradient. This requirement is nevertheless not necessarily very difficult to fulfil. The gas pressure typically falls $10 \%$ over one scale height in the radial direction, a value with which even subsonic turbulence can easily compete. The challenge is to have long-lived pressure enhancements, an issue which is still debated for the case of magnetorotational turbulence (Fromang \& Nelson 2006). Radial drift may also be significantly reduced in a dense boulder-dominated mid-plane layer where the gaseous headwind is reduced as the gas is dragged along with the boulders (Nakagawa et al. 1986).

The local absence of radial drift would not only solve the time-scale problem, but also reduce differential radial drift, which was shown by BDH to lead to destruction of the boulders due to collisions at speeds between 10 and $50 \mathrm{~m} / \mathrm{s}$ with slightly smaller boulders. The process of crossing from $1 \mathrm{~m}$ to $10 \mathrm{~m}$ would still be very inefficient, since the turbulent transport would cause $99 \%$ of the solid mass to be present in small fragments, but given that planet formation in our solar system took place over millions of years (see review by Trieloff \& Palme 2006), inefficient planetesimal formation may actually be required to comply with meteoritic evidence. Once a few extraordinarily lucky bodies cross the meter barrier, they could grow large enough to even sweep up the boulders still lying around in the mid-plane and thus continue to grow towards full-fledged protoplanets and later gas giant cores and terrestrial planets.

Acknowledgements. Part of this work was supported by the EU planets network. We are grateful to Jürgen Blum and Andrew Youdin for inspiring discussions on coagulation-fragmentation growth. We would like to thank the referee, Dr. Stuart Weidenschilling, for a thorough reading of the manuscript and for many useful suggestions for improvement.

\section{Appendix A: Stability analysis}

In this appendix we consider the stability of the equilibrium solution to Eq. (1). We find that the amplitude of any (arbitrarily large) perturbation to the equilibrium will decrease exponentially with time, so that Eq. (5) is a (both linearly and non-linearly) stable solution to the coagulation-fragmentation problem.

We start by rewriting Eq. (1) slightly by multiplying by $m_{1}$ and inserting $\rho_{1+2}=\rho_{1}+\rho_{2}$ (mass conservation) to avoid any reference to $\rho_{2}$. The resulting dynamical equation is

$$
\frac{\partial \rho_{1}}{\partial t}=-\rho_{1} \frac{\rho_{1+2}-\rho_{1}}{m_{2}} \sigma_{12} v_{12}+\frac{\left(\rho_{1+2}-\rho_{1}\right)^{2}}{m_{2}} \sigma_{22} v_{22} \text {. }
$$

We linearise this equation by writing the density of fragments $\rho_{1}$ as

$\rho_{1}=\bar{\rho}_{1}+\rho_{1}^{\prime}(t)$

where $\bar{\rho}_{1}$ is the equilibrium solution to Eq. (A.1),

$\bar{\rho}_{1}=\rho_{1+2} \frac{\sigma_{22} v_{22}}{\sigma_{12} v_{12}+\sigma_{22} v_{22}}$,

and $\rho_{1}^{\prime}$ is an infinitesimal perturbation to this equilibrium density. We ignored the other equilibrium solution, $\bar{\rho}_{1}=\rho_{1+2}$, because that state is not accompanied by any radius increase of the boulders (see discussion below). The expression in Eq. (A.3) is similar to Eq. (5), but we avoided approximating $\sigma_{22} \approx 4 \sigma_{12}$ for generality reasons. Inserting Eq. (A.2) into Eq. (A.1) yields the dynamical equation

$$
\begin{aligned}
\frac{\partial \rho_{1}^{\prime}}{\partial t}= & -\left(\bar{\rho}_{1}+\rho_{1}^{\prime}\right) \frac{\rho_{1+2}-\bar{\rho}_{1}-\rho_{1}^{\prime}}{m_{2}} \sigma_{12} v_{12} \\
& +\frac{\left(\rho_{1+2}-\bar{\rho}_{1}-\rho_{1}^{\prime}\right)^{2}}{m_{2}} \sigma_{22} v_{22}
\end{aligned}
$$

for the density perturbation $\rho_{1}^{\prime}$. Using the fact that $\bar{\rho}_{1}$ in itself satisfies Eq. (A.1) and ignoring terms of second order in the perturbed density yields the linearised dynamical equation

$$
\begin{aligned}
\frac{\partial \rho_{1}^{\prime}}{\partial t}= & -\frac{\sigma_{12} v_{12}}{m_{2}}\left(-2 \bar{\rho}_{1} \rho_{1}^{\prime}+\rho_{1}^{\prime} \rho_{1+2}\right) \\
& +\frac{\sigma_{22} v_{22}}{m_{2}}\left(-2 \rho_{1+2} \rho_{1}^{\prime}+2 \bar{\rho}_{1} \rho_{1}^{\prime}\right)
\end{aligned}
$$




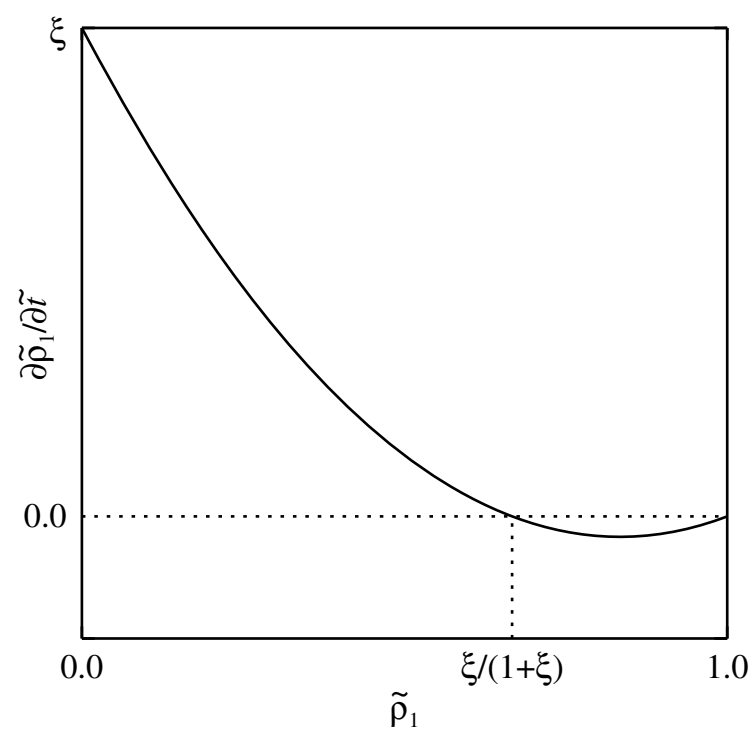

Fig. A.1. The dependence of the time derivative of the normalised density of fragments, $\partial \tilde{\rho}_{1} / \partial \tilde{t}$, on the density, $\tilde{\rho}_{1} \equiv \rho_{1} / \rho_{1+2}$, following Eq. (A.8). All states tend towards the equilibrium state at $\tilde{\rho}_{1}=\xi /(1+\xi)$. Here $\xi \equiv \sigma_{22} v_{22} /\left(\sigma_{12} v_{12}\right)$ is a parameter that depends on the collision speeds and on the cross section of the boulders. The equilibrium at $\tilde{\rho}_{1}=1$ is clearly unstable.

Now inserting the equilibrium solution $\bar{\rho}_{1}$ from Eq. (A.3) gives, after some trivial algebraic manipulation, the final equation for the evolution of the perturbed density as

$\frac{\partial \rho_{1}^{\prime}}{\partial t}=-\frac{\rho_{1+2}}{m_{2}} \sigma_{12} v_{12} \rho_{1}^{\prime}$.

Thus any small variation from the equilibrium expression will decay exponentially (following $\rho_{1}^{\prime}(t) \propto \exp (-\omega t)$ ) at the rate

$\omega=\frac{\rho_{1+2} \sigma_{12} v_{12}}{m_{2}}$.

The corresponding decay time $m_{2} /\left(\rho_{1+2} \sigma_{12} v_{12}\right)$ is, not surprisingly, similar to the time-scale of the sweep-up process, but with the the density of the fragments replaced by the total density $\rho_{1+2}$.

One may suspect that the equilibrium solution is also nonlinearly stable, due to the simple nature of Eq. (A.1). Rewriting Eq. (A.1) in terms of the normalised density $\tilde{\rho}_{1}=\rho_{1} / \rho_{1+2}$, normalised time $\tilde{t}=t /\left[m_{2} /\left(\rho_{1+2} \sigma_{12} v_{12}\right)\right]$ and collision parameter $\xi=\sigma_{22} v_{22} /\left(\sigma_{12} v_{12}\right)$ yields the evolution equation

$\frac{\partial \tilde{\rho}_{1}}{\partial \tilde{t}}=(1+\xi) \tilde{\rho}_{1}^{2}+(-1-2 \xi) \tilde{\rho}_{1}+\xi$

The second order polynomial $\partial \tilde{\rho}_{1} / \partial \tilde{t}=0$ has the solutions

$r_{1}=1$,

$r_{2}=\frac{\xi}{1+\xi}$.

In the first solution all the solid mass is bound in fragments - the total absence of boulders for this case means that coagulationfragmentation growth is disabled. The second solution is the same as in Eq. (A.3). Since we know that the polynomial in Eq. (A.8) opens upwards and that there is only one crossing of zero in the interval $\left[0,1\left[\right.\right.$, then all states of $\tilde{\rho}_{1}$ in this interval must approach the equilibrium solution given in Eq. (A.10). See Fig. A.1 for an illustration. The same arguments also show that $\tilde{\rho}_{1}=1$ (Eq. (A.9)) is an unstable solution, since even a vanishingly low number density of boulders will send the state towards $r_{2}$ instead. One must nevertheless still require some minimum number of boulders in the system for the continuity description of their number density to hold.

\section{Appendix B: Drag force}

In this appendix we describe the implementation of drag forces in our simulations. We let gas exert drag on the boulders following an Epstein drag law that is linear in the velocity difference between particles and gas. The gas velocity at the position of a particle is interpolated from the 27 nearest grid points using spline interpolation (see Youdin \& Johansen 2007). The Epstein drag law, with the friction time $\tau_{\mathrm{f}}$ given by

$\Omega_{\mathrm{K}} \tau_{\mathrm{f}}^{(\mathrm{Ep})}=\frac{a_{2}}{H} \frac{\rho_{\bullet}}{\rho_{\mathrm{g}}}$

is valid as long as the particle radius $a_{2}<(9 / 4) \lambda$, where $\lambda$ is the mean free path of the gas molecules. At $a_{2}>(9 / 4) \lambda$ one needs to use instead the Stokes friction time, given by $\Omega_{\mathrm{K}} \tau_{\mathrm{f}}^{(\mathrm{St})}=$ $\Omega_{\mathrm{K}} \tau_{\mathrm{f}}^{(\mathrm{Ep})} \times(4 / 9)\left(a_{2} / \lambda\right)$. The mean free path of the gas molecules can be calculated from

$\lambda=\frac{\mu}{\rho_{\mathrm{g}} \sigma_{\mathrm{mol}}}=\frac{\sqrt{2 \pi} \mu H}{\sum_{\mathrm{g}} \sigma_{\mathrm{mol}}}$,

where $\mu=3.9 \times 10^{-24} \mathrm{~g}$ is the mean molecular weight and $\sigma_{\text {mol }}=2.0 \times 10^{-15} \mathrm{~cm}^{2}$ is the cross section of molecular hydrogen (Chapman \& Cowling 1970; Nakagawa et al. 1986). In units of the gas scale height $H$ the mean free path can be expressed as

$\frac{\lambda}{H}=\frac{4.9 \times 10^{-9} \mathrm{~g} \mathrm{~cm}^{-2}}{\Sigma_{\mathrm{g}}}$

Assuming a minimum mass solar nebula model at $r=5 \mathrm{AU}$ we have $\Sigma_{\mathrm{g}}=150 \mathrm{~g} \mathrm{~cm}^{-2}$ and therefore a mean free path of $\lambda / H=3.3 \times 10^{-11}$. The transition from Epstein to Stokes regime thus occurs at $a_{2} / H=7.3 \times 10^{-11}$. We start our boulders with radius $a_{2} / H=10^{-11}$ (with $\Omega_{\mathrm{K}} \tau_{\mathrm{f}}=1$ ), so the Epstein regime is valid up to $\Omega_{\mathrm{K}} \tau_{\mathrm{f}} \approx 7.3$, which is already on the other side of the meter barrier. Since the focus of this paper is the crossing of the meter barrier, we shall for simplicity model drag force in the Epstein regime throughout and ignore the transition to the Stokes regime. The coagulation-fragmentation equilibrium has no dependence on the assumed amount of gas in the disc - the ratio of dust fragments to boulders depends only on the collision speeds (Eq. (5)), and while the radius growth (Eq. (6)) does scale with $\rho_{1+2}$, and thus with $\rho_{\mathrm{g}}$ if the solids-to-gas ratio is unchanged, the evolution of the Stokes number, St $\propto a_{2} / \rho_{\mathrm{g}}$, is independent of the gas density.

Another complication in modelling the Stokes regime is that the gas flow in the vicinity of a boulder would bring small dust grains around the boulder rather than onto its surface (Sekiya \& Takeda 2003). Dust grains may however still be able to penetrate to the boulder if the boulder is porous and has gas flow through it (Wurm et al. 2004). We shall nevertheless limit ourselves to the Epstein regime in this paper and leave the treatment of the interaction of grains and boulders in the Stokes regime to a future publication. 


\section{References}

Balbus, S. A., \& Hawley, J. F. 1991, ApJ, 376, 21

Balbus, S. A., \& Hawley, J. F. 1998, Rev. Mod. Phys., 70, 1

Balbus, S. A., \& Papaloizou, J. C. B. 1999, ApJ, 521, 650

Balbus, S. A., Hawley, J. F., \& Stone, J. M. 1996, ApJ, 467, 76

Beckwith, S. V. W., Sargent, A. I., Chini, R. S., \& Guesten, R. 1990, AJ, 99, 924

Benz, W. 2000, Space Sci. Rev., 92, 279

Bertout, C., Basri, G., \& Bouvier, J. 1988, ApJ, 330, 350

Blum, J., \& Wurm, G. 2000, Icarus, 143, 138

Bouwman, J., Lawson, W. A., Dominik, C., et al. 2006, ApJ, 653, L57

Brandenburg, A. 2003, in Advances in nonlinear dynamos, ed. A. Ferriz-Mas, \& M. Núñez (Taylor \& Francis), 269

Brandenburg, A., Nordlund, Å., Stein, R. F., \& Torkelsson, U. 1995, ApJ, 446, 741

Brauer, F., Dullemond, C. P., Johansen, A., et al. 2007, A\&A, 469, 1169

Brauer, F., Dullemond, C. P., \& Henning, Th. 2008, A\&A, 480, 859

Carballido, A., Fromang, S., \& Papaloizou, J. 2006, MNRAS, 373, 1633

Chapman, S., \& Cowling, T. G. 1970, The mathematical theory of non-uniform gases. an account of the kinetic theory of viscosity, thermal conduction and diffusion in gases (Cambridge: University Press), 3rd edn.

Chokshi, A., Tielens, A. G. G. M., \& Hollenbach, D. 1993, ApJ, 407, 806

Dominik, C., \& Tielens, A. G. G. M. 1997, ApJ, 480, 647

Dullemond, C. P., \& Dominik, C. 2004, A\&A, 421, 1075

Dullemond, C. P., \& Dominik, C. 2005, A\&A, 434, 971

Eisner, J. A., Chiang, E. I., \& Hillenbrand, L. A. 2006, ApJ, 637, L133

Fromang, S., \& Nelson, R. P. 2005, MNRAS, 364, L81

Fromang, S., \& Nelson, R. P. 2006, A\&A, 457, 343

Gammie, C. F. 1996, ApJ, 457, 355

Gammie, C. F. 2001, ApJ, 553, 174

Gullbring, E., Hartmann, L., Briceno, C., \& Calvet, N. 1998, ApJ, 492, 323

Hawley, J. F., Gammie, C. F., \& Balbus, S. A. 1995, ApJ, 440, 742

Henning, T., Dullemond, C. P., Wolf, S., \& Dominik, C. 2006, in Planet Formation, ed. H. Klahr, \& W. Brandner (Cambridge University Press), 112 Johansen, A., Klahr, H., \& Henning, Th. 2006, ApJ, 636, 1121

Johansen, A., Oishi, J. S., Low, M.-M. M., et al. 2007, Nature, 448, 1022

Klahr, H., \& Bodenheimer, P. 2003, ApJ, 582, 869

Lodato, G., \& Rice, W. K. M. 2004, MNRAS, 351, 630

Lommen, D., Wright, C. M., Maddison, S. T., et al. 2007, A\&A, 462, 211
Mizuno, H., Markiewicz, W. J., \& Voelk, H. J. 1988, A\&A, 195, 183

Nakagawa, Y., Sekiya, M., \& Hayashi, C. 1986, Icarus, 67, 375

Oishi, J. S., Mac Low, M.-M., \& Menou, K. 2007, ApJ, 670, 805

Pringle, J. E. 1981, ARA\&A, 19, 137

Rettig, T., Brittain, S., Simon, T., et al. 2006, ApJ, 646, 342

Rice, W. K. M., Lodato, G., Pringle, J. E., Armitage, P. J., \& Bonnell, I. A. 2004, MNRAS, 355, 543

Rodmann, J., Henning, T., Chandler, C. J., Mundy, L. G., \& Wilner, D. J. 2006, A\&A, 446, 211

Ryu, D., \& Goodman, J. 1992, ApJ, 388, 438

Safronov, V. S. 1969, Evoliutsiia doplanetnogo oblaka (English transl.: Evolution

of the Protoplanetary Cloud and Formation of Earth and the Planets, NASA Tech. Transl. F-677, Jerusalem: Israel Sci. Transl. 1972)

Sano, T., Miyama, S. M., Umebayashi, T., \& Nakano, T. 2000, ApJ, 543, 486

Schäfer, C., Speith, R., \& Kley, W. 2007, A\&A, 470, 733

Sekiya, M., \& Takeda, H. 2003, Earth, Planets, and Space, 55, 263

Semenov, D., Wiebe, D., \& Henning, T. 2004, A\&A, 417, 93

Shakura, N. I., \& Sunyaev, R. A. 1973, A\&A, 24, 337

Sicilia-Aguilar, A., Hartmann, L. W., Briceño, C., Muzerolle, J., \& Calvet, N. 2004, AJ, 128, 805

Suttner, G., \& Yorke, H. W. 2001, ApJ, 551, 461

Takeuchi, T., \& Lin, D. N. C. 2002, ApJ, 581, 1344

Testi, L., Natta, A., Shepherd, D. S., \& Wilner, D. J. 2003, A\&A, 403, 323

Trieloff, M., \& Palme, H. 2006, in Planet Formation, ed. H. Klahr, \& W. Brandner (Cambridge University Press), 64

Völk, H. J., Morfill, G. E., Roeser, S., \& Jones, F. C. 1980, A\&A, 85, 316

Weidenschilling, S. J. 1977, MNRAS, 180, 57

Weidenschilling, S. J. 1984, Icarus, 60, 553

Weidenschilling, S. J. 1997, Icarus, 127, 290

Weidenschilling, S. J., \& Cuzzi, J. N. 1993, in Protostars and Planets III, ed.

E. H. Levy, \& J. I. Lunine (University of Arizona Press), 1031

Whipple, F. L. 1972, in From Plasma to Planet, ed. A. Elvius (Wiley), 211

Wilner, D. J., Ho, P. T. P., Kastner, J. H., \& Rodríguez, L. F. 2000, ApJ, 534, L101

Wurm, G., Blum, J., \& Colwell, J. E. 2001, Icarus, 151, 318

Wurm, G., Paraskov, G., \& Krauss, O. 2004, ApJ, 606, 983

Wurm, G., Paraskov, G., \& Krauss, O. 2005, Icarus, 178, 253

Youdin, A., \& Johansen, A. 2007, ApJ, 662, 613

Youdin, A. N., \& Goodman, J. 2005, ApJ, 620, 459

Youdin, A. N., \& Lithwick, Y. 2007, Icarus, 192, 588 